\title{
Reduction of Vibrations of Passively-Isolated Ultra-Precision Manufacturing Machines using Mode Coupling
}

\author{
Jihyun Lee, Chinedum E. Okwudire \\ Mechatronics and Sustainability Research Laboratory \\ Department of Mechanical Engineering, University of Michigan
}

2350 Hayward, Ann Arbor, MI 48109

\begin{abstract}
Ultra-precision manufacturing (UPM) machines are used to fabricate and measure complex parts having micrometer-level features and nanometer-level tolerances/surface finishes. Therefore, random vibrations of the machine due to ground excitations and residual vibrations stemming from onboard disturbances must be mitigated using vibration isolation systems. A long-standing rule of thumb in vibration isolation system design is to locate the isolators in such a way that the vibration modes of the isolated machine are decoupled. However, prior work by the authors has demonstrated that coupling vibration modes of passively-isolated UPM machines could provide conditions for drastic reduction of residual vibrations compared to decoupling. The authors' analysis was based on the restrictive assumption that the isolated machine was modally damped. The key contribution of this paper is in investigating the effect of mode coupling on the residual vibrations of UPM machines with non-proportional (NP) damping - which is more realistic than modal damping. It also analyzes the effects of mode coupling on the reduction of ground vibrations (i.e., transmissibility). The analyses reveal that, even though NP damping


changes the vibration behavior of the machine compared to modal damping, mode coupling still provides ample opportunities to reduce residual vibrations and transmissibility. Guidelines for properly designing a UPM machine to best exploit mode coupling for vibration reduction are provided and validated through simulations and experiments. Up to $40 \%$ reduction in residual vibrations and $50 \%$ reduction in transmissibility are demonstrated.

Keywords: precision machine design, vibration isolation, residual vibrations, transmissibility

\section{Introduction}

Ultra-precision manufacturing (UPM) machines are designed to fabricate and measure complex parts having micrometer-level features and nanometer-level tolerances/surface finishes [1]. Examples of UPM machines include ultra-precision machine tools, wafer steppers and micro CMMs, to name a few. Vibrations of UPM machines must be mitigated, otherwise the stringent accuracy requirements of the machines cannot be achieved. A UPM machine may vibrate due to external excitations (mainly from the ground [2-5]) or its vibrations may be caused by onboard disturbances, e.g., the inertial reactions induced by moving machine components [2, 3, 6, 7]. Passive isolators provide an energy-neutral, reliable and cost-effective means for reducing ground excitations (i.e., transmissibility) but the soft mounting provided by such isolators leads to higher residual vibrations from onboard disturbances [6]. Design effort for passive isolators is therefore often focused on minimizing the tradeoff between residual vibrations and transmissibility $[6,8,9]$. 
A long-standing rule-of-thumb in isolation system design, recommended in academic literature and industrial practice, is to decouple all vibration modes of the isolated machine by aligning the isolator mounting locations with the center of gravity of the machine $[4,10-$ 14]. There are two important reasons for this rule. First, decoupling ensures that vertical ground motions are not transmitted to the typically more-sensitive horizontal axes of the machine. This is because, generally, vertical ground motions are more severe than horizontal ground motions [3, 4]. Secondly, decoupling ensures that two or more resonance peaks are not created in the transmissibility response of the machine, thereby reducing the rate of attenuation after the first resonance [13]. Even though it is very challenging in practice to locate the isolators exactly at the CG [3], UPM machine designers strive for this ideal, for example, by supporting the machine in a cradle structure or by focalizing the vibration isolators $[4,11,13,14]$.

There is anecdotal evidence scattered in the literature that suggests that coupling vibration modes by locating isolators away from the CG could help improve the response of passively-isolated UPM machines in terms of residual vibration (e.g., [2, 3]) and transmissibility (e.g., [15]). However, a good understanding of why and under what circumstances mode coupling is beneficial to UPM vibration reduction has been missing. Recent work by the authors [16-19] has sought to fill this knowledge gap. Of particular relevance to this paper is Ref. [16] where the authors showed that coupling vibration modes by properly selecting the location of the isolators could lead to the drastic reduction of residual vibrations in part due to a phenomenon known as mode localization [20-25]. When mode localization occurs, small changes in a system parameters can cause large variations 
in its mode shapes, leading to drastic changes in its response [24]. In studying mode localization (and a related phenomenon known as curve veering [26]), it is customary to ignore the effects of damping [21-23] or to assume modal damping [27]. However, the response of a non-proportionally damped system under coupling can be quite different from an undamped or modally damped system, as recently demonstrated by Vijayan and Woodhouse [28]. (Balmes [27] also alludes to this fact). The authors' prior work analyzing the effects of mode coupling on the residual vibrations of passively-isolated UPM machines [16] was based on the simplifying assumption of modal damping. Moreover, the study [16] did not analyze the effects of mode coupling on transmissibility. Therefore, building on some preliminary work by the authors $[29,30]$ the goals of this paper are to: (1) characterize the effect of mode coupling on the residual vibrations and transmissibility of a non-proportionally damped UPM machine; and (2) provide design guidelines for properly exploiting mode coupling for the reduction of residual vibrations and transmissibility.

The rest of this paper is organized as follows. Section 2 presents a planar model of a passively-isolated machine having NP damping and proposes frequency response functions (FRFs) for assessing the machine's residual vibrations and transmissibility. A perturbation method is used in Sections 3 to study the gradient or curvature of the $H_{\infty} / H_{2}$ norms of the residual vibrations and transmissibility FRFs, respectively, subject to mode coupling. The magnitude and direction of the gradient are used to deduce conditions under which mode coupling is most advantageous relative to decoupling, and the effects of NP damping on the results are highlighted. Section 4 provides practical design guidelines for judiciously placing vibration isolators to achieve reductions of residual vibration and transmissibility 
using mode coupling. In Section 5, the simulation and experimental studies conducted on a 5-axis ultra-precision milling machine in Ref. [16] are re-evaluated based on the NP damping analysis presented in this paper. Simulations and experiments are also carried out using a prototype UPM machine to further demonstrate the benefits and limitations of the analyses presented in this paper. Finally, conclusions and future work are presented in Section 6.

\section{Machine Model and Frequency Response Functions for Analyzing Vibrations}

\subsection{Machine Model}

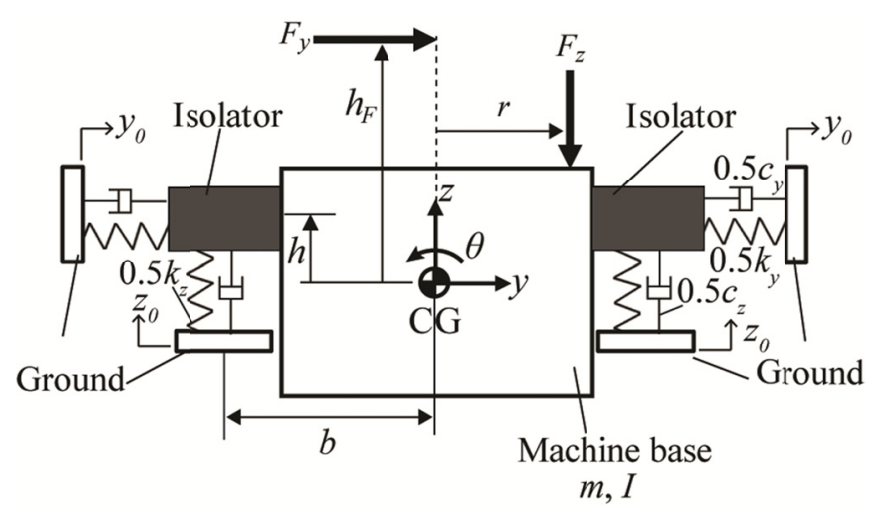

Figure 1: Planar model of an isolated machine

Figure 1 shows a 2-D model of an isolated machine. $m$ and $I$ are respectively the mass and centroidal moment of inertia of the machine base about the $x$-axis. $k_{y}, k_{z}$ and $c_{y}, c_{z}$ are the collective stiffness and damping constants of the passive isolators in the $y$ and $z$ directions, respectively. $b$ is the half-span of the isolators while $h$ is the vertical height of the isolator mounting point, measured from the center of gravity $(\mathrm{CG})$ of the base. The vibrations of the machine base are assumed to occur only in the $y$-z plane. Such planar analyses can be applied, for instance, when the machine's structure and loading are 
symmetrical in the $x$-direction. In general, the dynamics of passive (typically pneumatic) isolators is nonlinear $[31,32]$. However, for small vibratory motions, linear models are adequate [31].

Let $F_{y}$ and $F_{z}$ (shown in Figure 1) represent onboard disturbance forces applied at distances $h_{F}$ and $r$, respectively, from the machine's CG, and let $y_{0}$ and $z_{0}$ represent the horizontal and vertical displacements of the ground. The equation of motion of the isolated base is given by

$$
\mathbf{M u ̈}+\mathbf{C} \dot{\mathbf{u}}+\mathbf{K u}=\mathbf{F}+\mathbf{C}_{\mathbf{0}}+\mathbf{K u} \mathbf{u}_{0}
$$

$\mathbf{M}, \mathbf{C}, \mathbf{K}$ are respectively the mass, damping, stiffness matrices, while $\mathbf{u}, \mathbf{u}_{0}$ and $\mathbf{F}$ are respectively vectors of generalized displacements, ground displacements and generalized forces of the system. They are given by

$$
\begin{gathered}
\mathbf{M}=\left[\begin{array}{ccc}
m & 0 & 0 \\
0 & m & 0 \\
0 & 0 & I
\end{array}\right] ; \mathbf{C}=\left[\begin{array}{ccc}
c_{y} & 0 & -c_{y} h \\
0 & c_{z} & 0 \\
-c_{y} h & 0 & c_{\theta}+c_{y} h^{2}
\end{array}\right] ; \mathbf{K}=\left[\begin{array}{ccc}
k_{y} & 0 & -k_{y} h \\
0 & k_{z} & 0 \\
-k_{y} h & 0 & k_{\theta}+k_{y} h^{2}
\end{array}\right] ; \\
\mathbf{u}=\left\{\begin{array}{l}
y \\
z \\
\theta
\end{array}\right\} ; \mathbf{u}_{0}=\left\{\begin{array}{l}
y_{0} \\
z_{0} \\
0
\end{array}\right\} ; \mathbf{F}=\left[\begin{array}{cc}
1 & 0 \\
0 & -1 \\
-h_{F} & -r
\end{array}\right]\left[\begin{array}{c}
F_{y} \\
F_{z}
\end{array}\right]
\end{gathered}
$$

where $k_{\theta}=b^{2} k_{z}$ and $c_{\theta}=b^{2} c_{z}$. Note that the system's damping is explicitly considered in Eq.(1), unlike in Ref. [16] where the damping was ignored. As can be inferred from Eq.(2), only the dynamics in the $y$ and $\theta$ directions are coupled as a result of $h$. Therefore the effect of $h$ on the dynamics of the isolated system can be studied by focusing on the coupled subsystem 


$$
\begin{gathered}
\mathbf{M}=\left[\begin{array}{cc}
m & 0 \\
0 & I
\end{array}\right] ; \mathbf{C}=\left[\begin{array}{cc}
c_{y} & -c_{y} h \\
-c_{y} h & c_{\theta}+c_{y} h^{2}
\end{array}\right] ; \mathbf{K}=\left[\begin{array}{cc}
k_{y} & -k_{y} h \\
-k_{y} h & k_{\theta}+k_{y} h^{2}
\end{array}\right] ; \\
\mathbf{u}=\left\{\begin{array}{l}
y \\
\theta
\end{array}\right\} ; \mathbf{u}_{\mathbf{0}}=\left\{\begin{array}{c}
y_{0} \\
0
\end{array}\right\} ; \mathbf{F}=\left[\begin{array}{cc}
1 & 0 \\
-h_{F} & -r
\end{array}\right]\left[\begin{array}{c}
F_{y} \\
F_{z}
\end{array}\right]
\end{gathered}
$$

Equation (3) gives rise to two vibration modes, described by the coordinates $y$ and $\theta$, which are coupled by non-zero values of $h$. The effect of this coupled dynamics on residual vibrations and transmissibility is the subject of the rest of the paper.

\subsection{FRFs for Residual Vibrations}

Residual vibrations are transient in nature; they occur freely after $F_{y}$ or $F_{z}$ are removed and are typically quantified using time domain characteristics like settling time and maximum overshoot $[33,34]$. However, frequency domain metrics based on steady state assumptions often correlate closely with time domain characteristics [33, 35], while being more convenient for analysis. Therefore, in this paper, residual vibrations are quantified using FRFs.

Let us consider the effects of $F_{y}$ and $F_{z}$ on residual vibrations separately. When $F_{y}$ is applied to the machine, it generates responses in the $y$ and $\theta$ directions. Our previous analysis [16] was focused on the $\theta$ response (or rocking vibration) because it often dominates the residual vibration dynamics of UPM machines [2, 3]. However, in this paper, we take a more general approach by considering the weighted sum of both responses. i.e.,

$$
\left|H_{F y}\right|=\left|H_{y, F y}\right|+w\left|H_{\theta, F y}\right|
$$

where $w$ is a user-defined weighting factor having the dimension of length, while $H_{y, F y}$ and $H_{\theta, F y}$ are respectively the FRFs from force input $F_{y}$ to the $y$ and $\theta$ accelerations of the machine base. Note that, for a decoupled machine, the weight $w$ can be viewed as the 
absolute value of the height of a specific vibration-sensitive point above or below the CG. In practice, such a point could be a sensor, workpiece or tool location, depending on the application. We have assumed that $w=1 \mathrm{~m}$ in the rest of this paper in order to equally emphasize the rotational and translational dynamics of the machine.

The FRFs $H_{y, F y}$ and $H_{\theta, F y}$ of Eq.(4) can be expressed as

$$
\begin{gathered}
H_{y, F y}(\omega)=\frac{\ddot{y}}{F_{y}}=\frac{1}{m} \sum_{i=1}^{2}\left(A_{y i} X_{i}-B_{y i}\right) h_{N i} \\
H_{\theta, F y}(\omega)=\frac{\ddot{\theta}}{F_{y}}=\frac{1}{m \rho} \sum_{i=1}^{2}\left(A_{y i} X_{i}-B_{y i}\right)
\end{gathered}
$$

where $\rho$ is the centroidal radius of gyration of the isolated machine, while $A_{y i}, B_{y i}$ and $X_{i}$ are dimensionless functions pertaining to each mode $i(i=1,2)$. They are given by

$$
\begin{gathered}
A_{y i}=\frac{\tilde{h}_{F}-h_{N i}}{I_{q i}} ; X_{i}=\frac{\omega^{2}}{-\omega^{2}+2 j \omega \zeta_{y} \omega_{n i}^{2}+\omega_{n i}^{2}} ; \\
B_{y i}=\frac{X_{i}}{I_{q i}} \frac{2 j \varepsilon \beta \omega\left(A_{y 1} X_{1}+A_{y 2} X_{2}\right)}{2 j \varepsilon \beta \omega\left(X_{1} / I_{q 1}+X_{2} / I_{q 2}\right)+\omega^{2}}
\end{gathered}
$$

The variable $\omega$ represents dimensionless excitation frequencies and $j$ is the unit imaginary number. $\tilde{h}_{F}=h_{F} / \rho$ is the dimensionless forcing location of $F_{y}$, while $\omega_{n i}, h_{N i}$ and $I_{q i}$ respectively denote the dimensionless natural frequency, node height and modal inertia of each vibration mode $i$. Their definitions are provided in the appendix. The variable $\beta$ is given by

$$
\beta=\zeta_{\theta}-\varepsilon \zeta_{y}
$$

where 


$$
\zeta_{y}=\frac{c_{y}}{2 \sqrt{k_{y} m}} ; \zeta_{\theta}=\frac{c_{\theta}}{2 \sqrt{k_{\theta} I}} ; \varepsilon=\sqrt{\frac{k_{\theta}}{I} \frac{m}{k_{y}}}
$$

Similarly, the influence of $F_{z}$ on residual vibration can be characterized by accelerations in the $z$ and $\theta$ directions (for $r \neq 0$ ). However, as mentioned in Section 2.1, the motions in the $z$ direction are not of interest in this study because they are unaffected by $h$. Therefore, the residual vibration FRF in the $\theta$ direction is suitably described by

$$
H_{F z}(\omega)=\frac{\ddot{\theta}}{F_{z}}=\frac{1}{m \rho} \sum_{i=1}^{2}\left(A_{z i} X_{i}-B_{z i}\right)
$$

$X_{i}$ is defined in Eq.(7), while $A_{z i}$ and $B_{z i}$ are expressed as

$$
A_{z i}=\frac{\tilde{r}}{I_{q i}} ; \quad B_{z i}=\frac{X_{i}}{I_{q i}} \frac{2 j \varepsilon \beta \omega\left(A_{z 1} X_{1}+A_{z 2} X_{2}\right)}{2 j \varepsilon \beta \omega\left(X_{1} / I_{q 1}+X_{2} / I_{q 2}\right)+\omega^{2}}
$$

$\tilde{r}=r / \rho$ is the dimensionless version of $r$.

\subsection{FRF for Transmissibility}

It was mentioned in Section 1 that the requirement to decouple vibration modes in UPM machines was based on concerns related to transmissibility. The key reason being that coupling could make vertical ground motions to be transmitted to the typically moresensitive horizontal axes of the machine $[3,4]$. While this observation is generally true about coupling, it is not an issue if coupling is achieved by changing $h$ because the vertical motion is not coupled by $h$. In other words, transmissibility in the vertical direction is theoretically unaffected by changing $h$ [4]. Therefore, we focus our attention on transmissibility in the horizontal direction, and specifically, we consider the transmissibility between ground motion $y_{0}$ and $y_{w}$, the horizontal motion at height $h_{w}$ measured vertically from the CG (similar to $h_{F}$ in Figure 1). It is given by the expression 


$$
H_{t r}(\omega)=\frac{y_{w}}{y_{0}}=\sum_{i=1}^{2}\left(A_{t r i} X_{t r i}-B_{t r i}\right)\left(h_{N i}-\tilde{h}_{w}\right)
$$

$h_{w}$ (or its dimensionless form $\tilde{h}_{w}=h_{w} / \rho$ ) represents a sensitive location on the machine base. It could be, for instance, the location of the workpiece. $A_{t r i}, B_{t r i}$ and $X_{t r i}$ are defined as

$$
\begin{aligned}
& A_{t r i}=\frac{h_{N i}-\tilde{h}}{I_{q i}} ; \quad X_{t r i}=\frac{1+2 j \zeta_{y} \omega}{-\omega^{2}+2 j \omega \zeta_{y} \omega_{n i}^{2}+\omega_{n i}^{2}} ; \\
& B_{t r i}=\frac{X_{i}}{I_{q i}} \frac{2 j \varepsilon \beta \omega\left(A_{t r 1} X_{1}+A_{t r 2} X_{2}\right)}{2 j \varepsilon \beta \omega\left(X_{1} / I_{q 1}+X_{2} / I_{q 2}\right)+1}
\end{aligned}
$$

where $\tilde{h}=h / \rho$ is the dimensionless isolator height.

It is important, at this juncture, to explain the important role $\beta$ defined in Eq.(8) plays in the dynamics of the isolated system with NP damping. The decoupled system (with $h=$ 0) is always proportionally damped because each motion direction acts independently [36]; however, the coupled system can be proportionally or non-proportionally damped depending on the value of $\beta$. If $\beta=0$, the $B$ terms in Eqs. (5), (6), (10) and (12) equal zero, so the coupled system dynamics is proportionally damped, making it suitable for the modal damping assumption employed by the authors in Ref. [16]. For $\beta$ to be zero, the relationship $\zeta_{\theta} / \zeta_{y}=\varepsilon$ must be satisfied, which is scarcely achieved in practice. In most cases, therefore, $\beta$ $\neq 0$ so the system dynamics is non-proportionally damped. The implication is that as the system is coupled by changing $h$, it transitions suddenly from proportional to NP damping. At the same time, $\omega_{n i}, h_{N i}$ and $I_{q i}$ experience drastic changes linked to curve veering and mode localization, as discussed in Ref. [16] (also see appendix for summary). The next section explores how these different changes combine together to influence the residual vibration and transmissibility of the coupled system compared to the decoupled. 


\section{Analysis of Residual Vibration and Transmissibility of Mode-Coupled UPM Machines}

The steady state responses of vibrating systems are often quantified in frequency domain using either $H_{2}$ or $H_{\infty}$ norms of FRFs [37, 38]. A system's $H_{2}$ norm is a measure of the energy of its response to stationary random excitations. It is thus well suited for quantifying the effect of the transmissibility of a UPM machine over a wide band of

frequencies $[5,12]$. The $H_{\infty}$ norm is its worst case steady-state response to sinusoidal inputs, represented by the peak magnitude of its FRF. Both norms are useful indicators of the system's response to transient vibrations [35]. In Ref. [16], the authors adopted the output variance (which at its limit is equivalent to the $H_{2}$ norm [37]) as a measure of the system's residual vibration response because it permits the effects of all resonance modes in an FRF to be represented by a single function. However, the output variance is sensitive to the anti-resonances as well as the frequency limits of the FRF, which are often not key contributors to the system's transient response. Therefore, for quantifying residual vibrations in this paper, the $H_{\infty}$ norm is preferred because it focuses on the highest resonance peak in the FRF, which often correlates well with the dominant frequency in the transient vibration response. In this section, the $H_{\infty}$ norms of the residual vibration FRFs and output variance of the transmissibility FRF are analyzed, using a perturbation technique, to understand the effects mode coupling on UPM machines with NP damping.

\subsection{Residual Vibration}

The $H_{\infty}$ norm of residual vibrations due to $F_{y}$ (i.e., $H_{F y}$ defined in Eq.(4)) is expressed as 


$$
\left\|H_{F y}\right\|_{\infty}=\max \left(\left|H_{y, F y}\left(\omega_{y}\right)\right|,\left|H_{\theta, F y}\left(\omega_{\theta}\right)\right|\right)
$$

where $\omega_{y}$ and $\omega_{\theta}$ are the respective resonance frequencies of $H_{y, F y}$ and $H_{\theta, F y}$. At $h=0$, they are given by

$$
\omega_{y}=\frac{1}{\sqrt{1-2 \zeta_{y}^{2}}} ; \quad \omega_{\theta}=\frac{\varepsilon}{\sqrt{1-2 \zeta_{\theta}^{2}}}
$$

We investigate the effect of mode coupling on $\left\|H_{F y}\right\|_{\infty}$ by evaluating the first-order Taylor expansion of $\left|H_{y, F y}\left(\omega_{y}\right)\right|$ and $\left|H_{\theta, F y}\left(\omega_{\theta}\right)\right|$ about $h=0$. i.e.,

$$
\left|H_{y, F y}\left(\omega_{y}\right)\right| \cong C_{y, F y}\left(1+T_{y} \tilde{h}\right)
$$

and

$$
\left|H_{\theta, F y}\left(\omega_{\theta}\right)\right| \cong C_{\theta, F y}\left(1+T_{\theta} \tilde{h}\right)
$$

$C_{y, F y}$ and $C_{\theta, F y}$ represent the values of $\left|H_{y, F y}\left(\omega_{y}\right)\right|$ and $\left|H_{\theta, F y}\left(\omega_{\theta}\right)\right|$ at $h=0$. They are given by

$$
C_{y, F y}=\frac{1}{m} \frac{1}{2 \zeta_{y} \sqrt{1-\zeta_{y}^{2}}} ; \quad C_{\theta, F y}=\frac{1}{m \rho} \frac{\left|\tilde{h}_{F}\right|}{2 \zeta_{\theta} \sqrt{1-\zeta_{\theta}^{2}}}
$$

$T_{y}$ and $T_{\theta}$ are the corresponding gradients at $h=0$ expressed as

$$
T_{y}=\frac{-\tilde{h}_{F}\left(\varepsilon^{2}+\left(4 \varepsilon \zeta_{y} \zeta_{\theta}-1\right) \omega_{y}^{2}\right)}{\left(\left(\varepsilon^{2}-\omega_{y}{ }^{2}\right)^{2}+4 \omega_{y}{ }^{2} \varepsilon^{2} \zeta_{\theta}^{2}\right)} ; T_{\theta}=\frac{-\left(1+\left(4 \zeta_{y}^{2}-1\right) \omega_{\theta}^{2}\right)}{\tilde{h}_{F}\left(\left(1-\omega_{\theta}{ }^{2}\right)^{2}+4 \omega_{\theta}{ }^{2} \zeta_{y}^{2}\right)}
$$

The dominant peak of $\left|H_{F y}\right|$ (i.e., $\left\|H_{F y}\right\|_{\infty}$ ) at $h=0$ is determined by evaluating the ratio $w C_{\theta, F y} / C_{y, F y} ;$ i.e.,

$$
\frac{w C_{\theta, F y}}{C_{y, F y}}=\left|\frac{w h_{F}}{\rho^{2}}\right| \frac{\zeta_{y} \sqrt{1-\zeta_{y}^{2}}}{\zeta_{\theta} \sqrt{1-\zeta_{\theta}^{2}}}
$$


If the ratio is greater than unity, the rocking vibration dominates the response of the decoupled system and $\left|H_{\theta, F y}\left(\omega_{\theta}\right)\right|$ should be the focus of the analysis. Otherwise, the translatory vibration is dominant and the analysis is focused on $\left|H_{y, F y}\left(\omega_{y}\right)\right|$. From Eq. (20), one observes that the rocking vibration dominates when $\zeta_{\theta}$ is small relative to $\zeta_{y}$ or $h_{F}$ is large relative to $\rho^{2}$ (for a given $w$ ).

The gradients, $T_{y}$ and $T_{\theta}$, provide useful hints about the effects of small perturbations of $h($ from $h=0$ ) on the dominant residual vibration response of the coupled system. In mode localization literature, coupling with small values of $h$ is referred to as "weak coupling" [22]. The magnitude of the gradient indicates the sensitivity of the decoupled system to weak coupling while its direction shows whether residual vibrations can be expected to increase or decrease due to weak coupling.

The direction of each gradient can be determined by solving for the values of $\varepsilon$ where the gradient crosses zero. They are given by

$$
\varepsilon= \begin{cases}\frac{\sqrt{1-2 \zeta_{y}^{2}+4 \zeta_{y}^{2} \zeta_{\theta}^{2}}-2 \zeta_{y} \zeta_{\theta}}{1-2 \zeta_{y}^{2}} & \text { for } T_{y}=0 \\ \frac{\sqrt{1-2 \zeta_{\theta}^{2}}}{\sqrt{1-4 \zeta_{y}^{2}}} & \text { for } T_{\theta}=0\end{cases}
$$

Figure 2(a) and (b) provide graphical depictions of Eq.(21) for various values of $\varepsilon$ as functions $\zeta_{y}$ and $\zeta_{\theta}$ between 0 and $0.5 . h_{F}>0$ is assumed because it is common in practice to have moving masses above the CG of the machine. However, please keep in mind that if $h_{F}<0$, the signs of $T_{y}$ and $T_{\theta}$ must be reversed in the ensuing discussions. Observe that when $\varepsilon<1, T_{y}>0$ and $T_{\theta}<0$ for most combinations of $\zeta_{y}$ and $\zeta_{\theta}$. Conversely, when $\varepsilon>1$, 
$T_{y}<0$ and $T_{\theta}>0$ under most scenarios. The implication of these observations on isolation system design is discussed in Section 4.

Figure 3(a) and (b) show the $T_{y}$ and $T_{\theta}$ plots for the modally damped system. $\zeta_{1}$ and $\zeta_{2}$ are the modal damping ratios of the lower and higher frequency modes, respectively. When $\varepsilon<1, \zeta_{1}=\zeta_{\theta}$ and $\zeta_{2}=\zeta_{y}$. The opposite is true for $\varepsilon>1$. As can be seen from the plots, the general conclusions reached for the NP damped system apply to the modally damped system. The behavior of the signs of $T_{y}$ and $T_{\theta}$ are consistent with the changes in the system's mode shapes as the system transitions from $\varepsilon<1$ to $\varepsilon>1$ (see $h_{N i}$ plot in appendix and detailed discussion in Ref.[16]). Notice, however, that the pattern of the contours in Fig. 3 is different from those in Fig. 2 due to the effect of NP damping.

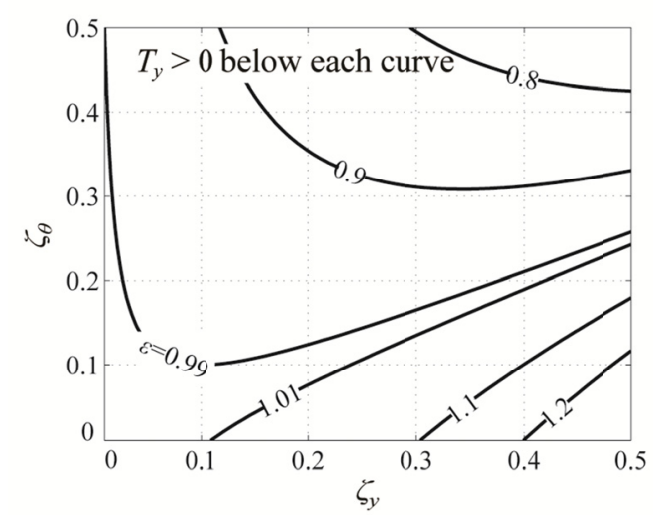

(a)

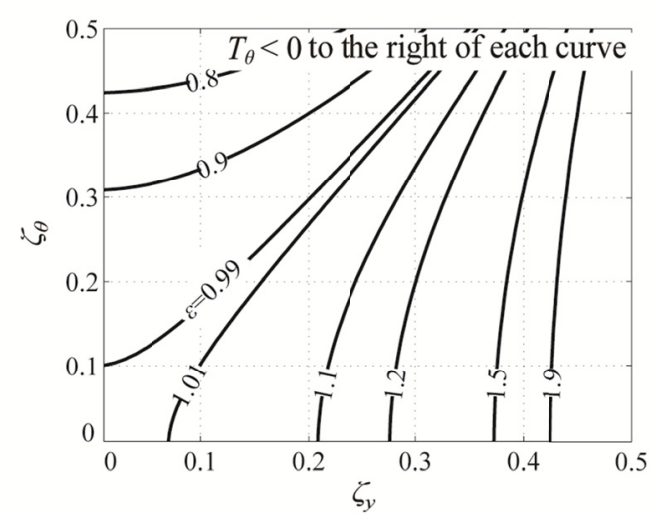

(b)

Figure 2: Contour plots of (a) $T_{y}=0$ (b) $T_{\theta}=0$ of NP damped system as functions of $\zeta_{y}$ and $\zeta_{\theta}$ for various values of $\varepsilon$. $h_{F}>0$ is assumed 


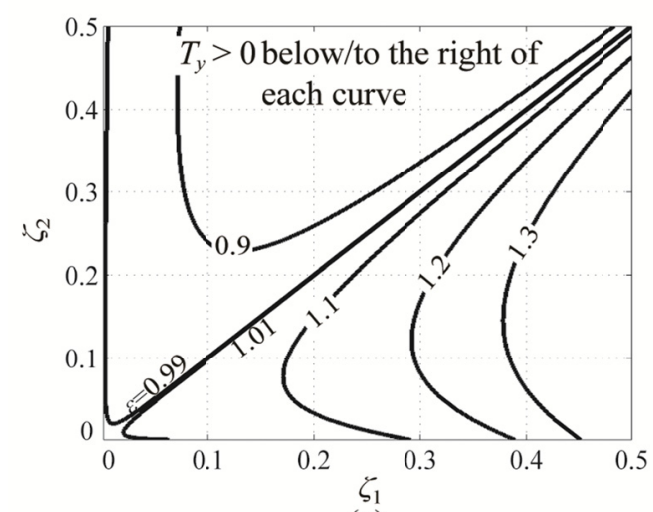

(a)

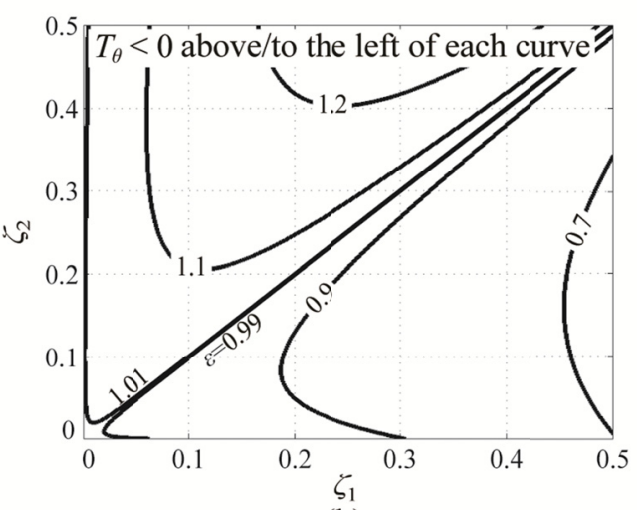

(b)

Figure 3: Contour plots of (a) $T_{y}=0$ (b) $T_{\theta}=0$ of modally damped system as functions of $\zeta_{1}$ and $\zeta_{2}$ for various values of $\varepsilon . h_{F}>0$ is assumed

The magnitude of the gradient is an indicator of how sensitive the residual vibration magnitude of the decoupled system is to weak coupling. It is helpful to know the conditions for maximum sensitivity in order to more fully exploit the beneficial effects of mode coupling in design. If modal damping is assumed, the gradients of $T_{y}$ and $T_{\theta}$ have their highest magnitude (which tends to infinity) as $\varepsilon \rightarrow 1$. It corresponds to the condition of maximal sensitivity of the system's mode shapes due to mode coupling [16] (also see $h_{N i}$ plot in appendix). However, when NP damping is considered, the transition of system's mode shapes from real to complex gives rise to very different conditions for maximum sensitivity to mode coupling. Let $\varepsilon^{*}$ and $\varepsilon^{*}{ }_{\theta}$ denote the $\varepsilon$ values where the respective magnitudes of $T_{y}$ and $T_{\theta}$ are highest, i.e.,

$$
\left.\frac{d T_{y}}{d \varepsilon}\right|_{\varepsilon_{\varepsilon=\varepsilon_{y}^{*}}^{*}}=0 ;\left.\frac{d T_{\theta}}{d \varepsilon}\right|_{\varepsilon_{\varepsilon=\varepsilon_{\theta}^{*}}^{*}}=0
$$

Obtaining $\varepsilon_{y}^{*}$ from Eq. (22) requires the solution of a fifth order polynomial, 


$$
\begin{aligned}
& \varepsilon^{5}+a_{4} \varepsilon^{4}+a_{3} \varepsilon^{3}+a_{2} \varepsilon^{2}+a_{1} \varepsilon+\left.a_{0}\right|_{\varepsilon=\varepsilon_{y}^{*}}=0 \\
& a_{4} \triangleq 6 \omega_{y}^{2} \zeta_{y} \zeta_{\theta}, a_{3} \triangleq-2 \omega_{y}^{2}, a_{2} \triangleq 4 \omega_{y}{ }^{4} \zeta_{\theta}\left(-1+2 \zeta_{\theta}^{2}\right) \zeta_{y}, \\
& a_{1} \triangleq \omega_{y}{ }^{4}\left(1-4 \zeta_{\theta}^{2}\right), a_{0} \triangleq-2 \omega_{y}{ }^{6} \zeta_{y} \zeta_{\theta}
\end{aligned}
$$

Figure 4(a) shows a sampling of the positive real values of $\varepsilon_{y}^{*}$ obtained by solving Eq. (23) numerically for $\zeta_{y}$ and $\zeta_{\theta}$ values ranging from 0 to 0.5 . Each root is tested numerically to ensure that it results in a local maximum. As seen, there is a wide range of $\varepsilon$ values that give rise to maximum sensitivity in $H_{y}$ due to weak coupling. Solving for $\varepsilon^{*}{ }_{\theta}$ from Eq. (22) yields two real roots which can be obtained in closed-form as

$$
\varepsilon_{\theta}^{*}=\frac{\sqrt{1-2 \zeta_{\theta}^{2}}}{\sqrt{1 \pm 2 \zeta_{y}}}
$$

Both roots result in local maxima in $\left|T_{\theta}\right|$. Figure 4(b) shows various values of $\varepsilon^{*}{ }_{\theta}$ as functions of the damping ratios $\zeta_{y}$ and $\zeta_{\theta}$. We observe that the smaller value of $\varepsilon^{*}{ }_{\theta}$ in Eq.(24) always yields a negative value for $T_{\theta}$ while the larger value results in positive $T_{\theta}$. Also, the smaller $\varepsilon^{*}{ }_{\theta}$ always results in a larger magnitude of $\left|T_{\theta}\right|$ than the larger $\varepsilon^{*}{ }_{\theta}$. Notice from Eq. (24) that, for non-zero damping ratios, $\varepsilon^{*}{ }_{\theta}=1$ only when $\zeta_{\theta}=\sqrt{ } \zeta_{y}$. One can therefore conclude that because of NP damping, the coupled system has finite sensitivities to mode coupling and that the maximum sensitivity does not necessarily occur as $\varepsilon \rightarrow 1$, where the mode shapes of the modally damped system are most sensitive. 


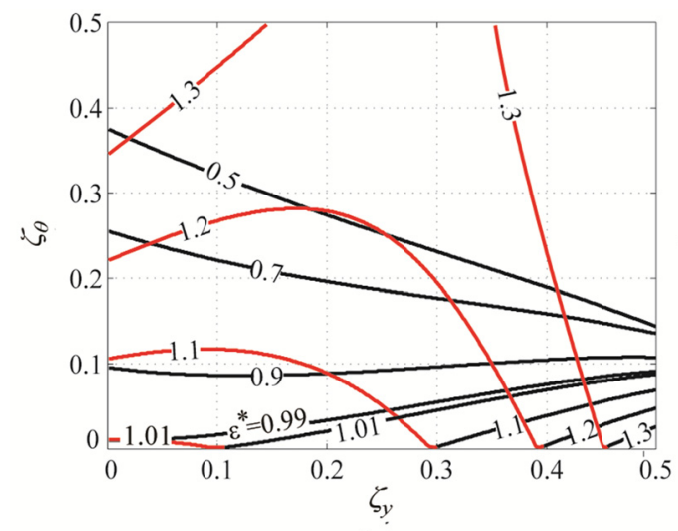

(a)

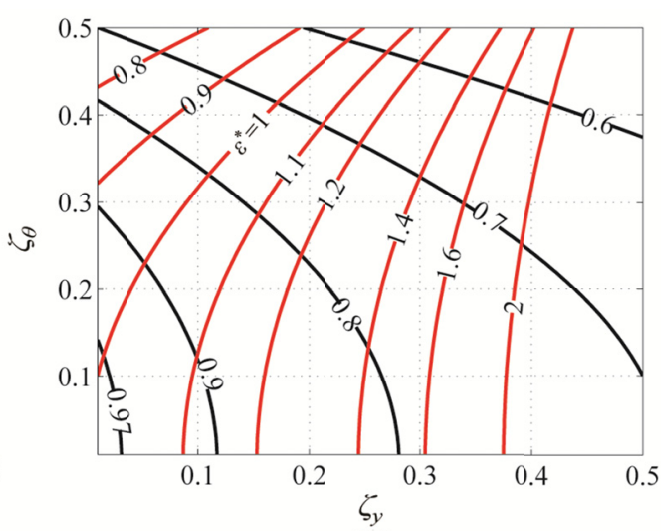

(b)

Figure 4: Contour plots of (a) $\varepsilon_{y}^{*}$ and (b) $\varepsilon_{\theta}^{*}$ as functions of $\zeta_{y}$ and $\zeta_{\theta}$. The red and black line colors are used to differentiate each of the two (positive real) roots of Eq.(22). All roots of Eq.(22) correspond to local maxima in $T_{y}$ or $T_{\theta}$

Considering residual vibrations due to $F_{z}$, the $H_{\infty}$ norm of $H_{F z}$ is given by

$$
\left\|H_{F z}\right\|_{\infty}=\left|H_{\theta, F z}\left(\omega_{\theta}\right)\right|
$$

where $\omega_{\theta}$ is the resonance frequency, defined in Eq. (15) for $h=0$. A second-order Taylor expansion is needed to evaluate $\left\|H_{F z}\right\|_{\infty}$ because the gradient term in its first-order expansion is always zero, meaning that it always has a stationary point at $h=0$ (as also observed in Ref.[16] for the modally damped system). Evaluating the second-order Taylor expansion of $\left\|H_{F z}\right\|_{\infty}$ about $h=0$, we get

$$
\left|H_{F z}\left(\omega_{\theta}\right)\right| \cong C_{F z}\left(1+\frac{1}{2} T_{z} \tilde{h}^{2}\right)
$$

The value of $\left\|H_{F z}\right\|_{\infty}$ at $h=0, C_{F z}$, is given by

$$
C_{F z}=\frac{1}{m \rho} \frac{|\tilde{r}|}{2 \zeta_{\theta} \sqrt{1-\zeta_{\theta}^{2}}}
$$

$T_{z}$ represents the curvature at $h=0$ expressed as 


$$
T_{z}=\frac{-2 \omega_{\theta}{ }^{2}\left[\left(4 \varepsilon \zeta_{\theta} \zeta_{y}-1+4 \zeta_{y}{ }^{2}\right) \omega_{\theta}{ }^{4}+\left(1+\varepsilon^{2}-4 \varepsilon^{2} \zeta_{y}{ }^{2}\right) \omega_{\theta}{ }^{2}-\varepsilon^{2}\right]}{\left[\omega_{\theta}{ }^{4}-2\left(1-2 \zeta_{\theta}{ }^{2}\right) \varepsilon^{2} \omega_{\theta}{ }^{2}+\varepsilon^{4}\right]\left[\omega_{\theta}{ }^{4}-2\left(1-2 \zeta_{y}{ }^{2}\right) \omega_{\theta}{ }^{2}+1\right]}
$$

The nature of the stationary point (i.e., local maximum, minimum or inflection point) at $h=0$ is determined by numerically evaluating the sign of $T_{z}$ for various combinations of $\zeta_{y}, \zeta_{\theta}$ and $\varepsilon$. Note that $\tilde{r}$ has no influence on $T_{z}$. Figure 5 shows the contour plot of $T_{z}=0$ for various values of $\varepsilon . T_{z}$ is negative for most combinations of $\varepsilon, \zeta_{y}$ and $\zeta_{\theta}$, meaning that $h=0$ is most often a local maximum of $\left\|H_{F z}\right\|_{\infty}$. The implication is that (weak) coupling of vibration modes by raising or lowering the isolators w.r.t. the CG is more often better than decoupling, in terms of reducing residual vibration caused by $F_{z}$. The same conclusion was reached in the analysis presented in Ref. [16]. However, much like the case of $\left\|H_{F y}\right\|_{\infty}$ discussed above, it can be observed that NP damping changes the pattern of the curves significantly compared to the modally damped case. Figure 6 (a) and (b) show the $T_{z}$ plots for the modally damped system when $\varepsilon<1$ and $\varepsilon>1 . \zeta_{1}$ and $\zeta_{2}$ are the same as described in the context of Figure 3.

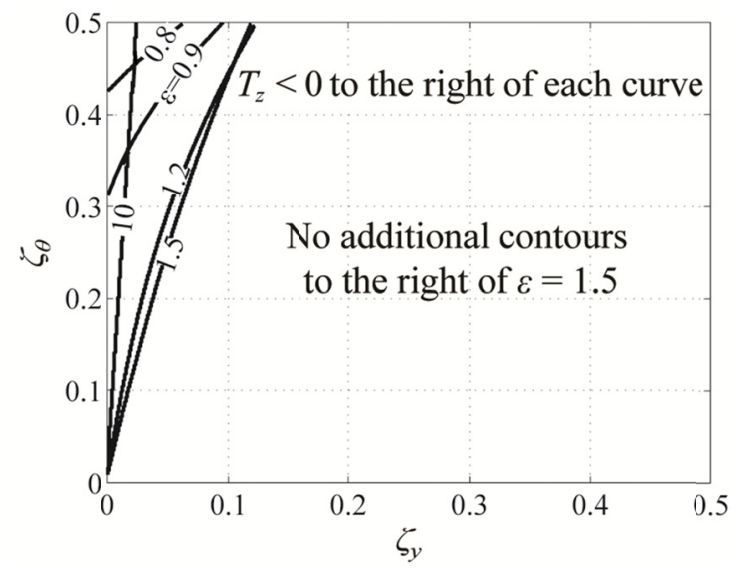

Figure 5: Contour plot of $T_{z}=0$ of NP damped system as functions of $\zeta_{y}$ and $\zeta_{\theta}$ for various values of $\varepsilon$ 


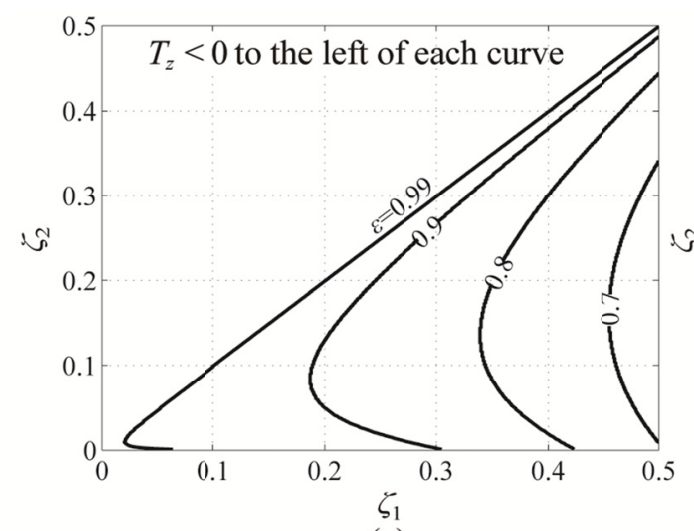

(a)

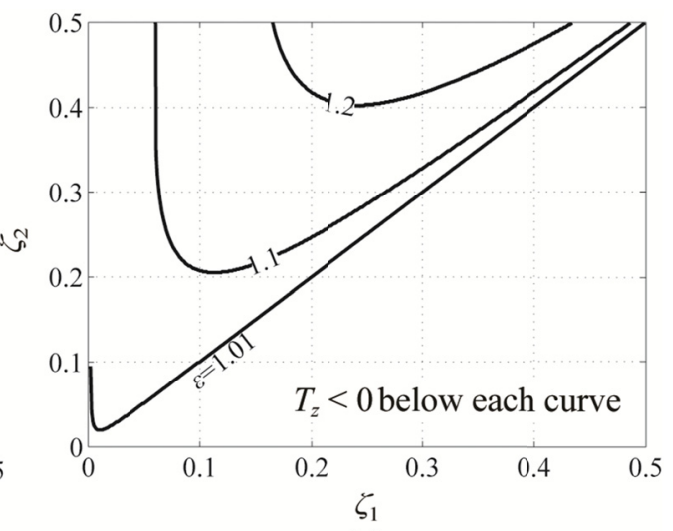

(b)

Figure 6: Contour plots of $T_{z}=0$ of modally damped system as functions of $\zeta_{1}$ and $\zeta_{2}$ for various values of (a) $\varepsilon<1$ (b) $\varepsilon>1$

To characterize the sensitivity of the system with NP damping to mode coupling, we determine $\varepsilon_{z}^{*}$, the $\varepsilon$ values where the magnitude of $T_{z}$ is highest, by solving the equation

$$
\left.\frac{d T_{z}}{d \varepsilon}\right|_{\varepsilon=\varepsilon_{z}^{*}}=0
$$

and selecting the solutions that yield local maxima. Obtaining $\varepsilon^{*}$ from Eq. (29) requires the solution of a fifth order polynomial,

$$
\begin{aligned}
& a_{5} \varepsilon^{5}+a_{4} \varepsilon^{4}+a_{3} \varepsilon^{3}+a_{2} \varepsilon^{2}+a_{1} \varepsilon+\left.a_{0}\right|_{\varepsilon=\varepsilon_{z}^{*}}=0 \\
& a_{5} \triangleq \zeta_{y}, a_{4} \triangleq-\zeta_{\theta}\left(1-4 \zeta_{y}^{2}\right), a_{3} \triangleq 2 \zeta_{y}\left(1-2 \zeta_{\theta}^{2}\right)\left(1-2 \zeta_{y}^{2}\right), \\
& a_{2} \triangleq 2 \zeta_{\theta}\left(1-2 \zeta_{\theta}^{2}\right), a_{1} \triangleq-3 \zeta_{y}\left(1-2 \zeta_{\theta}^{2}\right)^{2}, a_{0} \triangleq-\zeta_{\theta}\left(1-2 \zeta_{\theta}^{2}\right)^{2}
\end{aligned}
$$

Figure 7 shows a sampling of the positive real values of $\varepsilon^{*}{ }_{z}$ (that yield a local maxima) obtained by solving Eq.(30) numerically for $\zeta_{y}$ and $\zeta_{\theta}$ values ranging from 0 to 0.5 . Again, we see that the maximum sensitivity to coupling does not necessarily occur as $\varepsilon \rightarrow 1$, where the modally damped system is most sensitive. 


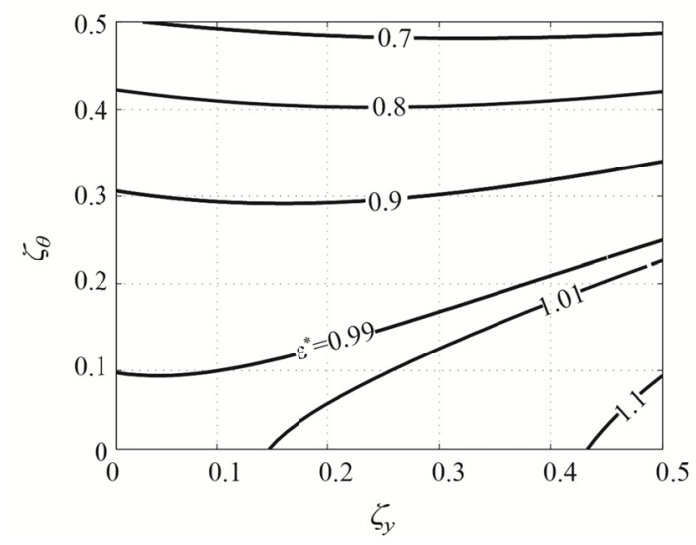

Figure 7: Contour plot of $\varepsilon^{*}$ as functions of $\zeta_{y}$ and $\zeta_{\theta}$, obtained by selecting the positive real roots of Eq.(29) that yield local maxima in $T_{z}$

\subsection{Transmissibility}

The output variance (or simply variance) of a UPM machine with transmissibility FRF $H_{t r}$ to a zero-mean white noise input of unit magnitude is given by [12]

$$
\sigma_{H}^{2}=\int_{0}^{\omega_{\max }}\left|H_{t r}(\omega)\right|^{2} d \omega
$$

The integration limit $\omega_{\max }$ is usually set to infinity. However, if the system's input is known, $\omega_{\max }$ can be taken as the highest frequency of its spectrum. The effect of weak coupling on transmissibility can be studied by analyzing the first-order Taylor expansion of $\sigma_{H}^{2}$ about $h=0$, given by the expression

$$
\sigma_{H}^{2} \cong C_{t r}\left(1+T_{t r} \tilde{h}\right)
$$

$C_{t r}$ and $T_{t r}$ represent the value and gradient of $\sigma_{H}^{2}$ at $h=0$, respectively. They are defined as

$$
C_{t r}=\int_{0}^{\omega_{\max }}\left|\mathrm{I}_{0}\right|^{2} d \omega ; \quad T_{t r}=\frac{2 h_{w}}{C_{t r}} \int_{0}^{\omega_{\max }} \mathrm{I}_{1} \cdot\left|\mathrm{I}_{0}\right|^{2} d \omega
$$

where $\mathrm{I}_{0}$ and $\mathrm{I}_{1}$ are given by 


$$
\mathrm{I}_{0}=\frac{1+j 2 \zeta_{y} \omega}{1-\omega^{2}+j 2 \zeta_{y} \omega} ; \mathrm{I}_{1}=\frac{\omega^{2}\left(\omega^{2}-\varepsilon^{2}\right)}{\left[\left(\omega^{2}-\varepsilon^{2}\right)^{2}+\left(2 \beta \varepsilon \omega+2 \varepsilon^{2} \omega \zeta_{y}\right)^{2}\right]}
$$

It is very challenging to calculate the integral for $T_{t r}$ in Eq.(33) analytically; therefore, it is evaluated numerically. To approximate the integration limit of infinity, $\omega_{\max }=100$ is selected - i.e., 100 times the horizontal natural frequency of the decoupled system (see definition of dimensionless $\omega$ in appendix). The direction of the gradient is determined by numerically solving for the values of $\varepsilon$ where each gradient crosses zero, using values of $\zeta_{y}$ and $\zeta_{\theta}$ over the interval $[0.01,0.5]$. The analysis reveals that $T_{t r}$ is always positive when $\varepsilon<$ 1. For $\varepsilon>1$, the sign of $T_{t r}$ can be determined from Figure 8 . The $\varepsilon$ value (i.e., $\varepsilon^{*}{ }_{t r}$ ) corresponding to the maximum sensitivity of $T_{t r}$ is obtained by investigating the roots of the equation

$$
\left.\frac{d T_{t r}}{d \varepsilon}\right|_{\varepsilon=\varepsilon_{t r}^{*}}=0
$$

which requires the following equation to be satisfied

$$
\left.\int_{0}^{\omega_{\max }} \mathrm{I}_{2} \cdot\left|\mathrm{I}_{0}\right|^{2} d \omega\right|_{\varepsilon=\varepsilon_{\text {tr }}^{*}}=0
$$

where $\mathrm{I}_{2}$ is given by

$$
\mathrm{I}_{2}=\frac{2 \varepsilon \tilde{h}_{w} \omega^{2}\left[\left(\omega^{2}-\varepsilon^{2}\right)^{2}-4 \varepsilon \omega^{2} \zeta_{y} \zeta_{\theta}\left(\omega^{2}-\varepsilon^{2}\right)-4 \omega^{4} \zeta_{\theta}^{2}\right]}{\left[\left(\omega^{2}-\varepsilon^{2}\right)^{2}+\left(2 \beta \varepsilon \omega+2 \varepsilon^{2} \omega \zeta_{y}\right)^{2}\right]^{2}}
$$

Figure 9 shows a sampling of $\varepsilon^{*}$ tr values obtained by solving Eq.(36) numerically and selecting the roots that yield local maxima in $T_{t r}$. As seen, there is a wide range of $\varepsilon$ values that give rise to maximum sensitivity in $H_{t r}$ due to weak coupling. 


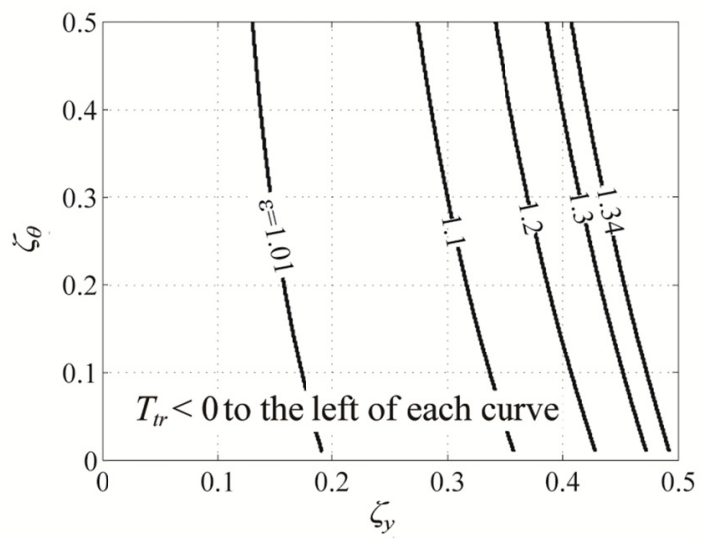

Figure 8: Contour plot of $T_{t r}=0$ of NP damped system as functions of $\zeta_{y}$ and $\zeta_{\theta}$ for various values of $\varepsilon . h_{w}>0$ is assumed

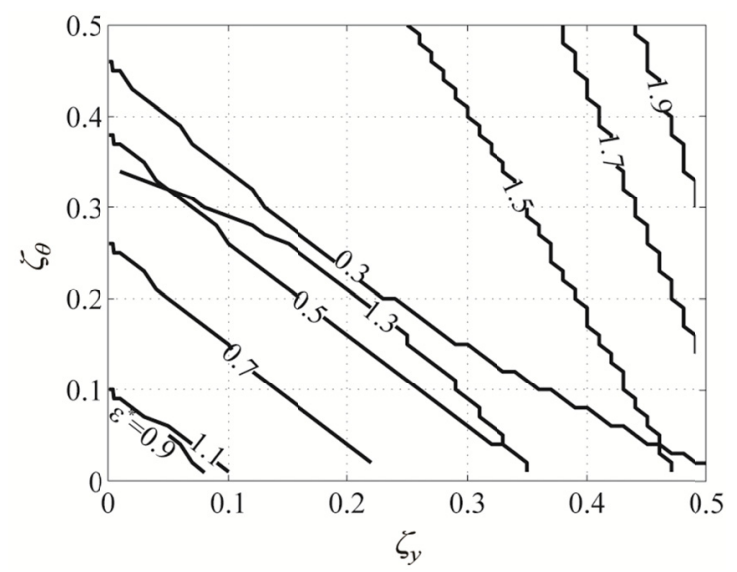

Figure 9: Contour plot of $\varepsilon^{*}$ tr as functions of $\zeta_{y}$ and $\zeta_{\theta}$

\section{Practical Design Guidelines Based on Analysis}

This section demonstrates how the results from the analyses of Section 3 can be used to provide hints to a designer on when and how to couple the vibration modes of a given UPM machine by changing $h$. Design considerations for residual vibration reduction are considered first followed by those for transmissibility reduction. The reader is encouraged to review the examples in Section 3.3 of Ref. [16] for more details on how to apply the proposed analyses in isolation system design for UPM machines. 


\subsection{Design Considerations for Residual Vibration Reduction}

Equation (20) combined with Figures 2 and 4 are useful tools for designing a UPM machine to reduce $\left\|H_{F y}\right\|_{\infty}$ via weak coupling. Using Eq.(20), one can determine which vibration mode $-y$ or $\theta$ - is dominant for the decoupled machine; the dominant mode can then become the focus of attention for residual vibration reduction. Armed with the knowledge of the dominant mode, Figure 2 provides hints on what direction to make small changes $h$ in order to reduce residual vibration. For instance if the $y$-mode is dominant, one can determine the sign of $T_{y}$ that corresponds to the $\varepsilon, \zeta_{y}$ and $\zeta_{\theta}$ of a given UPM machine. If $T_{y}<0$ then placing the isolators higher than the CG (i.e., positive $h$ ) is more desirable. The opposite is true of $T_{y}>0$. Similar inferences can be drawn if the $\theta$-mode is dominant by monitoring the sign of $T_{\theta}$. Figure 4 is useful in that it tells a designer which $\varepsilon, \zeta_{y}$ and $\zeta_{\theta}$ combinations would provide the greatest reduction of $\left\|H_{F y}\right\|_{\infty}$ through small changes in $h$. For instance, pneumatic isolators usually have a knob for adjusting $\zeta_{\theta}$ (by changing $c_{z}$ ). Given $\varepsilon$ and $\zeta_{y}$ for a specific UPM machine, a designer may want to tweak $\zeta_{\theta}$ so that the combination of $\varepsilon, \zeta_{y}$ and $\zeta_{\theta}$ for the machine lands on one of the curves of Figure 4.

Note that care has to be taken when $w C_{\theta} / C_{y}$ is close to unity due to the tendency to have opposite trends in the signs of $T_{y}$ and $T_{\theta}$ for a given $\varepsilon, \zeta_{y}$ and $\zeta_{\theta}$ combination (as can be seen by comparing Figure 2 (a) and (b)). The implication is that coupling may reduce the dominant peak of the decoupled system but at the same time increase the non-dominant peak such that it becomes dominant after coupling. Another point to remember is that the analysis of Section 3 is only valid for small changes in $h$. It does not provide any information on how much the CG can be lowered or raised before the residual vibrations 
begin to increase or the system becomes unstable. The analysis by Ryaboy [15] provides useful guidance for a designer with regard to stability limits. In addition, a designer may want to plot $\left\|H_{F y}\right\|_{\infty}$ as a function of $h$ to determine the limits of $h$ with regard to vibration reduction. To highlight why such a plot is important, let us assume that a UPM machine is designed for maximum sensitivity in the $\theta$ direction (i.e., $\varepsilon=\varepsilon^{*}{ }_{\theta 1}$, the smaller of $\varepsilon^{*}{ }_{\theta}$ in Eq.(24)). We would like to know the value of $|\tilde{h}|=\tilde{h}_{\max }$ beyond which $\left\|H_{F y}\right\|_{\infty}$ becomes higher than its original magnitude at $h=0$. In other words, $\tilde{h}_{\max }$ is the dimensionless height of the isolators beyond which the vibration reduction benefits achieved by mode coupling (relative to decoupling) are nullified. Figure 10 shows contour plots of $\tilde{h}_{\max }$ as functions of $\zeta_{y}$ and $\zeta_{\theta}$ for $\tilde{h}_{F}=0.5,1,1.5$ and 2 . The hatched portion of each plot represents the design space where coupling increases residual vibration relative to decoupling. The color spectrum indicates the relative magnitudes of $\tilde{h}_{\max }$. As one observes, the relative sizes of the various regions of each are slightly different for each $\tilde{h}_{F}$ value. In each case, however, there is a sizeable region where coupling remains more beneficial than decoupling for large values of $\tilde{h}_{\max }(>1.5)$. However, each plot also has a region where $\tilde{h}_{\max }$ values greater than about 0.4 nullify any benefits of coupling relative to decoupling.

Extending the design insights to $\left\|H_{F z}\right\|_{\infty}$, we observe that since $T_{z}<0$ in most scenarios, the decoupled state of the machine yields a local maximum of $\left\|H_{F z}\right\|_{\infty}$, except for the very small subset of the design space to the left of each $\varepsilon$ curve in Figure 5 where $\left\|H_{F z}\right\|_{\infty}$ has a local minimum at $h=0$. This result is very auspicious for residual vibration reduction in general using weak mode coupling because most of the conditions that reduce $\left\|H_{F y}\right\|_{\infty}$ also 
reduce $\left\|H_{F z}\right\|_{\infty}$. Therefore, a designer often does not have to tradeoff one for the other.

However, notice that the curves for $\varepsilon_{z}^{*}$ in Figure 7 are very different from those of $\varepsilon_{y}^{*}$ or $\varepsilon^{*} \theta$ in Figure 4. This means that a designer may have to choose between tuning their machine for maximum sensitivity in $\left\|H_{F y}\right\|_{\infty}$ or $\left\|H_{F z}\right\|_{\infty}$, depending on which they deem to be more critical or beneficial.
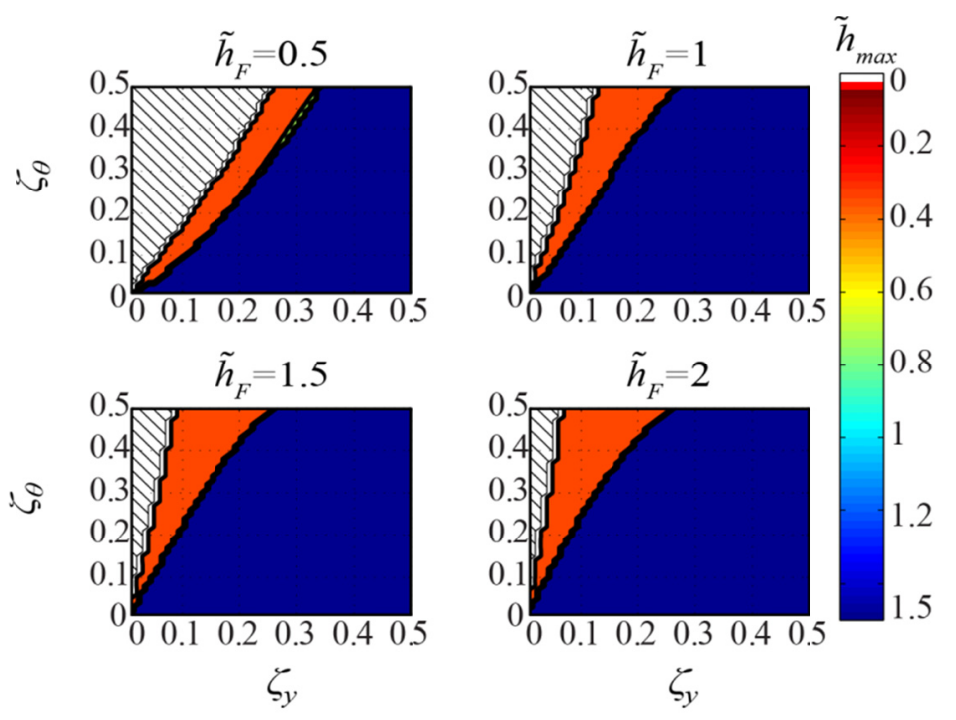

Figure 10: Contour plot of $\widetilde{\boldsymbol{h}}_{\text {max }}$ as functions of $\zeta_{y}$ and $\zeta_{\theta}$ for various values of $\widetilde{h}_{F}$. In this example, the system is assumed to be designed with $\varepsilon=\varepsilon^{*}{ }_{\theta 1}$. The hatched sections of each plot indicate regions where mode coupling increases vibrations

\subsection{Design Considerations for Transmissibility Reduction}

The logic used for determining design guidelines for residual vibration reduction can be applied to transmissibility. When $\varepsilon<1, T_{t r}>0$ always, meaning that weak coupling with negative $h$ is always better than decoupling in terms of transmissibility. We had also observed that for $\varepsilon<1, T_{y}>0$ for most combinations of $\zeta_{y}$ and $\zeta_{\theta}$ in Figure 2. This means that it is highly probable to simultaneously reduce transmissibility and residual vibration via weak coupling when $\varepsilon<1$. The broader implication is that vibration reduction using mode coupling is not necessarily constrained by the well-known tradeoff between 
transmissibility and residual vibration reduction in passive systems! For $\varepsilon>1$ the situation is not as straightforward; there is much less overlap between the conditions for reduction of residual vibration and transmissibility. However, a designer can use the directions and most sensitive conditions for $\left\|H_{F y}\right\|_{\infty},\left\|H_{F z}\right\|_{\infty}$ and $\sigma_{H}^{2}$ presented in this paper as a guide to locate $h$ (and tune $\varepsilon, \zeta_{y}$ or $\zeta_{\theta}$ ) so that a good compromise is struck between residual vibration and transmissibility reduction for the UPM machine.

\section{Simulation and Experimental Validation}

This section presents two sets of simulation and experiment results to validate the theoretical analysis presented in this paper. The first set revisits the simulation and experiment conducted in Ref. [16] on a 5-axis ultra-precision milling machine to highlight the importance of considering NP damping compared to modal damping. The machine used in Ref. [16] was a commercial UPM machine to which the authors no longer have access. Moreover, as reported in Ref. [16], there were practical limitations to the experiments that could be conducted on the machine. Therefore, a second set of simulations and laboratory experiments are conducted on a prototype of a UPM machine base which has been specially designed to remove many of the restrictions that may be presented by a specific commercial UPM machine.

\subsection{Simulation and Experiments on 5-Axis Milling Machine}

Figure 11 shows the schematic of the Mori Seiki's NN1000 ultra-precision 5-axis milling machine that was employed for the simulations and experiments in Ref. [16]. The machine is supported on four pneumatic vibration isolators and is symmetrical about its 
centroidal $x-z$ and $y-z$ planes, such that the 2-D model presented in this paper is applicable to both planes. However, as explained in Ref. [16], the residual vibrations in its $x-z$ plane were negligible because of the relatively small moving mass of its $x$-axis. Consequently, the simulations and experiments reported in Ref. [16] were focused on the $y$-z plane of the machine. Furthermore, transmissibility was not analyzed in the paper [16]. Therefore, only $\left\|H_{F y}\right\|_{\infty}$ and $\left\|H_{F z}\right\|_{\infty}$ are considered in this first set of simulations/experiments.

Considering $\left\|H_{F y}\right\|_{\infty}$, the parameters provided in Table 1 are used to calculate $\rho=285$ $\mathrm{mm}, \tilde{h}_{F}=1.53, \varepsilon=1.21, \zeta_{y}=\zeta_{\theta}=0.15$. Eq.(20) gives the ratio $C_{\theta, F y} / C_{y, F y}$ as 5.36, meaning that $H_{\theta, F y}\left(\omega_{\theta}\right)$ (i.e., the rocking vibration) is dominant when $h=0$. With $\varepsilon=1.21$ and $\zeta_{y}=\zeta_{\theta}$ $=0.15, T_{\theta}>0$ can be deduced from Figure 2(b). This means that placing the isolators below the $\mathrm{CG}$ (i.e., $h<0$ ) will reduce the resonance peak of $\left|H_{\theta, F y}\right|$. From Eq. (24), $\varepsilon^{*}{ }_{\theta}=0.86$ or $\varepsilon^{*} \theta$ $=1.17$ can be calculated. This means that, with $\varepsilon=1.21$, the machine is close to a condition for maximum sensitivity to weak coupling (i.e., $\varepsilon^{*}{ }_{\theta}=1.17$ ). Note, however, that $T_{y}<0$ at $\varepsilon$ $=1.21$, meaning that selecting $h<0$ will increase the peak of the less-dominant $\left|H_{y, F y}\right|$; but since $C_{\theta, F y} / C_{y, F y}$ is far from unity, the reduction in the peak of $\left|H_{\theta, F y}\right|$ can be expected to outweigh the increase in the peak of $\left|H_{y, F y}\right|$ for small changes in $h$. Weak coupling can therefore be expected to reduce residual vibrations compared to decoupling. The same conclusions were reached in Ref. [16] based on the analysis employing modal damping, and the residual vibrations of the machine were observed in experiments to drastically reduce when $h$ was changed from 0 to $-116 \mathrm{~mm}$, as shown in Figure 12. Figure 13 provides an explanation for the similarities between the modal and the NP damping predictions for the machine. As can be seen, the resonance peak of $\left|H_{\theta, F y}\right|$ for modal and NP damping have 
the same qualitative behavior in the interval $\tilde{h} \in[-0.41,0]$ (i.e. $h \in[-116,0] \mathrm{mm}$ ). It was mere coincidence that the analysis of Ref. [16] was conducted within this interval. However, the influence of NP damping can be observed in the slightly different sensitivities to weak coupling around $h=0$ - the magnitude of $T_{\theta}$ is a bit smaller with NP damping compared to the modally damped case. Moreover, even though the analysis presented in this paper is focused on the region around $h=0$, one observes that the influence of NP damping on the machine's vibration response is much more significant when $h>0$ and $h<-116 \mathrm{~mm}$. Also, the $h>0$ portion of the plot demonstrates that conclusions derived based on coupling in the vicinity of $h=0$ may not hold for larger values of $h$, hence we need to determine $\tilde{h}_{\max }$ for the particular condition of interest, as discussed in Section 4. Simulated and measured $\left|H_{\theta, F y}\right|$ plots for the machine are shown in Figure 14 (a) and (b). At $h=0$, the modally and NP damped systems have exactly the same response, which is reasonably close to that measured from the machine. At $h=-116 \mathrm{~mm}$ one observes that the peaks of the NP damped system (which differ slightly from those of the modally damped) are in better agreement with those of the measured response, as should be expected since $\beta \neq 0$ for the machine. 


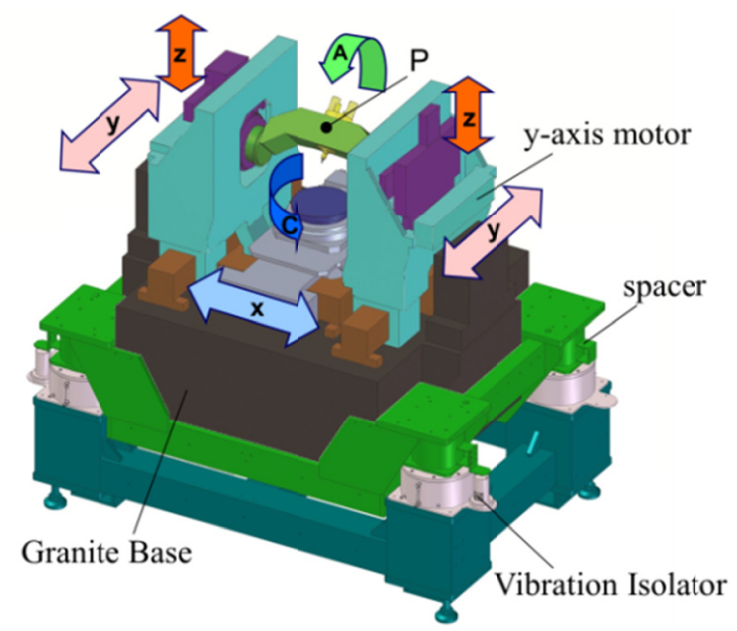

Figure 11: Schematic of Mori Seiki's NN1000 DCG 5-axis ultra-precision machine tool used for simulations and experiments

\begin{tabular}{|c|c|}
\hline Parameter & Value \\
\hline$m[\mathrm{~kg}]$ & 1,182 \\
\hline$I\left[\mathrm{~kg}-\mathrm{m}^{2}\right]$ & 96 \\
\hline$k_{y}[\mathrm{~N} / \mathrm{m}]$ & 880,000 \\
\hline$k_{\theta}[\mathrm{Nm} / \mathrm{rad}]$ & 104,430 \\
\hline$h_{F}[\mathrm{~mm}]$ & 435 \\
\hline$c_{y}[\mathrm{~kg} / \mathrm{s}]$ & 9676 \\
\hline$c_{\theta}[\mathrm{Nm}-\mathrm{s} / \mathrm{rad}]$ & 950 \\
\hline$r[\mathrm{~mm}]$ & 70 \\
\hline
\end{tabular}

Table 1: Key parameters of NN1000 DCG used for simulations 
(a)

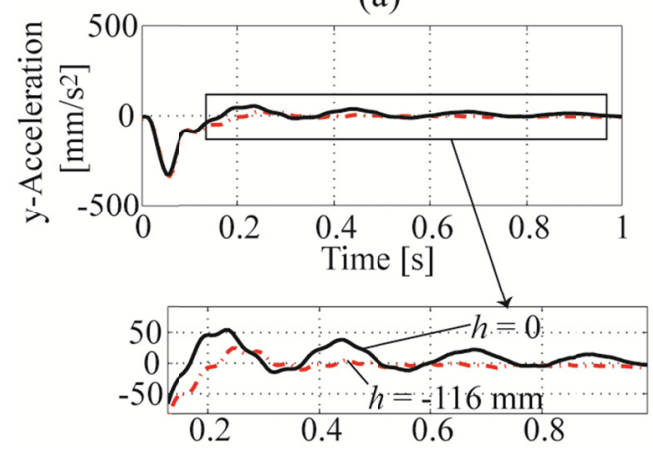

(b)

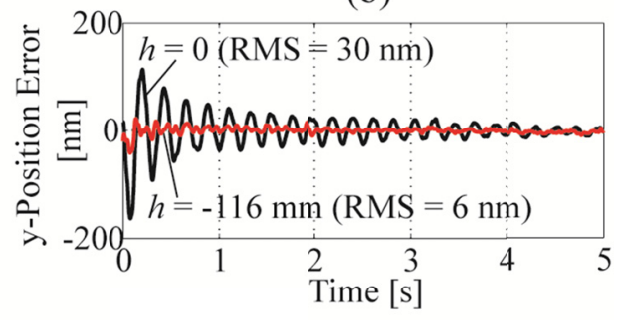

Figure 12: (a) Acceleration of base measured at point $P$ (b) position error measured from encoder for $h=0$ and $h=-116 \mathrm{~mm}$ during motion of $y$-Axis from rest to 1000 $\mathbf{m m} / \mathbf{m i n}$

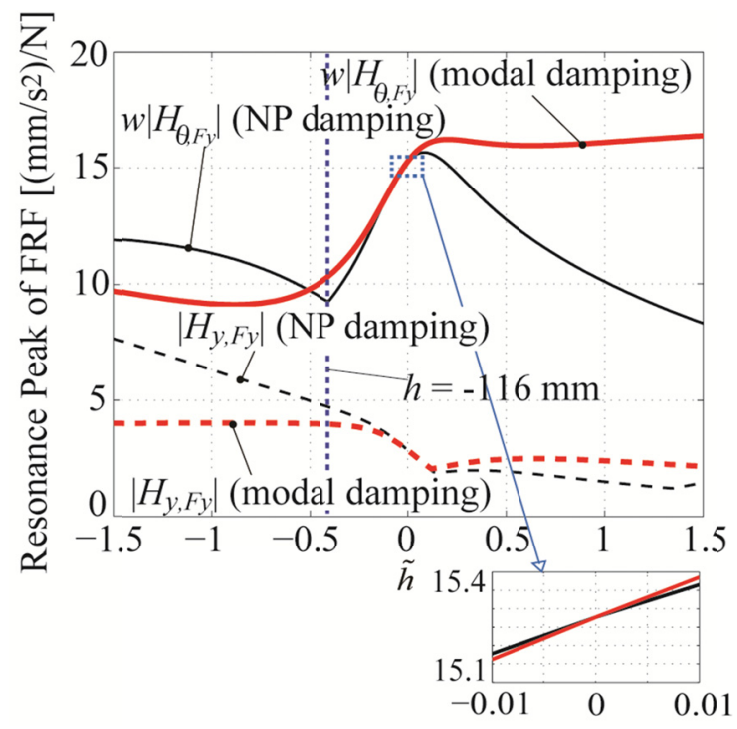

Figure 13: Resonance peaks of $\left|H_{y, F y}\right|$ and $w\left|H_{\theta, F y}\right|$ of NN1000 as functions of dimensionless isolator height. $w=1 \mathrm{~m}$. Note: $h=-116 \mathrm{~mm}$ corresponds to $\widetilde{h}=-0.41$

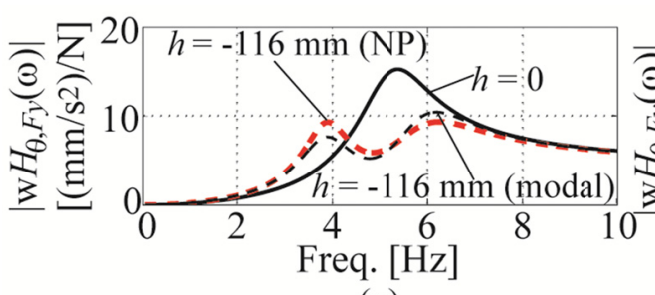

(a)

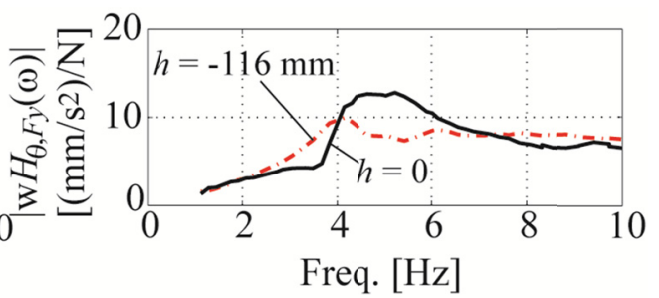

(b)

Figure 14: FRFs obtained from (a) simulations and (b) experiments for $h=0$ and $h=-116 \mathrm{~mm} . w=1 \mathrm{~m}$ 
$\left\|H_{F z}\right\|_{\infty}$ is investigated in simulation only because the vibrations induced by the $z$-axis could not be measured reliably in the NN1000 machine due to their relatively low levels, as reported in Ref. [16]. Using $\varepsilon=1.21$ and $\zeta_{y}=\zeta_{\theta}=0.15, T_{z}<0$ can be deduced from Figure 5 , which means that $\left\|H_{F z}\right\|_{\infty}$ decreases in either direction of $h$. Figure 15 provides a comparison between the modal and NP damping-based plots of $\left\|H_{F z}\right\|_{\infty}$. In the contrast with results of $\left\|H_{F y}\right\|_{\infty}$, the $\left\|H_{F z}\right\|_{\infty}$ of the NN 1000 machine is more sensitive to coupling with NP damping compared to modal damping. However, similar to $\left\|H_{F y}\right\|_{\infty}$, the influence of NP damping on the machine's vibration response becomes more pronounced as the system becomes more coupled. It is interesting to note that with $\varepsilon=1.21$, the machine is approximately three times less sensitive to weak coupling than with $\varepsilon_{z}^{*}=0.997\left(T_{z}=-23.24\right.$ and -8 , respectively, at $\varepsilon=0.977$ and 1.21 ). This means that a lot of value could be gained by tweaking the machine's parameters to coincide with $\varepsilon_{z}^{*}$, as shown in Figure 15.

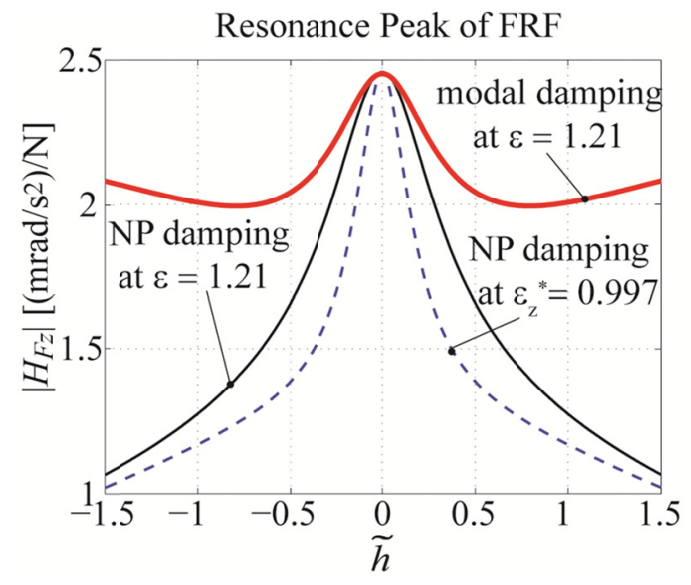

Figure 15: $\left|H_{F z}\right|$ of NN1000 as functions of dimensionless isolator height simulation and experiments on UPM machine base prototype 


\subsection{Simulation and Experiments on UPM Machine Base Prototype}

Figure 16 shows the reconfigurable UPM machine base prototype used for additional validating simulations/experiments in this section. The prototype consists of a 0.8 ton base of dimensions $749.3 \mathrm{~mm} \times 749.3 \mathrm{~mm} \times 495.3 \mathrm{~mm}$, supported symmetrically on a foundation by four pneumatic isolators (Bilz model BiAir 1-ED). The arrays of threaded holes on the base allow the isolators to be mounted at different discrete locations so that $h$ can be varied. For evaluating residual vibrations, an electromagnetic shaker (APS Dynamics model APS 113) is attached to the base and is used to provide the excitation forces $F_{y}$ and $F_{z}$ at locations $h_{F}$ and $r$. The vibration of the structure is measured using accelerometers (PCB Piezotronics model 393B05) with sensitivity of $10.42 \mathrm{~V} / \mathrm{g}$. Transmissibility experiments are conducted by using an impact hammer (PCB Piezotronics model 086D20) to excite the foundation in the horizontal direction; the horizontal accelerations of the foundation (i.e., $\ddot{y}_{0}$ ) and those of the base (at height $h_{w}$ from the CG) are measured and used to determine the transmissibility. Table 2 summarizes the relevant parameters of the prototype. The isolator stiffness and damping coefficients have been identified experimentally, while the mass and moment of inertia of the base have been determined from its $\mathrm{CAD}$ model. Its mass and inertia are reported as a range of values in the table due to slight variations resulting from the repositioning of the shaker (and balance masses to keep the base level) for each experiment. 


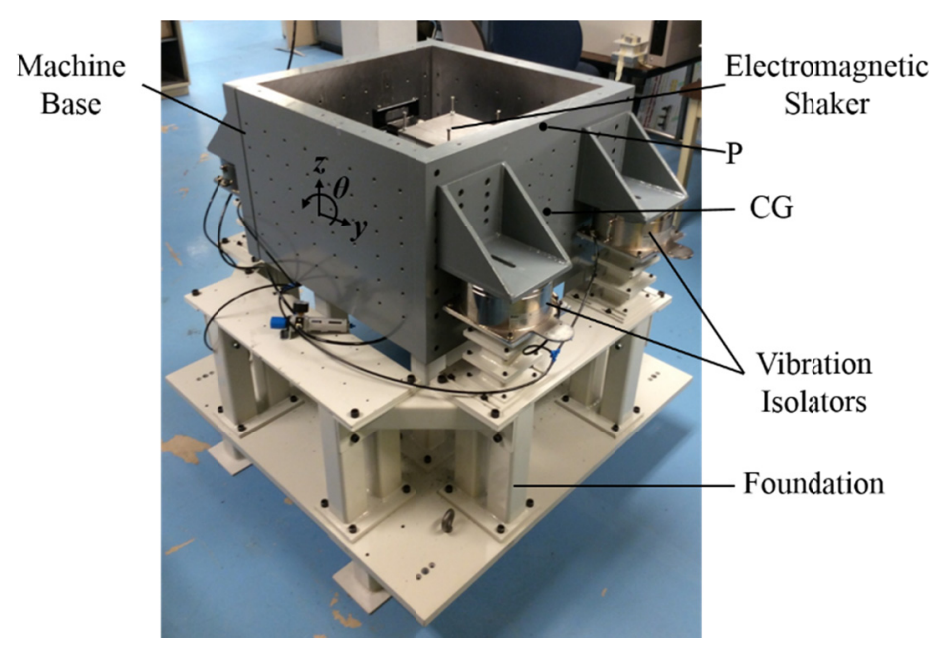

Figure 16: Reconfigurable UPM machine base prototype used for simulations and experiments. Note that the point labelled $P$ represents a sensitive location on the machine base (i.e., the distance between the $C G$ and point $P$ is $h_{w}$ )

\begin{tabular}{|c|c|}
\hline Parameter & Value \\
\hline$m[\mathrm{~kg}]$ & $830-834$ \\
\hline$I\left[\mathrm{~kg}-\mathrm{m}^{2}\right]$ & $77-80$ \\
\hline$k_{v}[\mathrm{~N} / \mathrm{m}]$ & $1,150,319$ \\
\hline$k_{\theta}[\mathrm{Nm} / \mathrm{rad}]$ & 80,289 \\
\hline$c_{y}[\mathrm{~N} \cdot \mathrm{s} / \mathrm{m}]$ & 3,739 \\
\hline$c_{\theta}[\mathrm{Nm}-\mathrm{s} / \mathrm{rad}]$ & 1,118 \\
\hline$h_{F}[\mathrm{~mm}]$ & 130 \\
\hline$r[\mathrm{~mm}]$ & 119 \\
\hline$h_{w}[\mathrm{~mm}]$ & 220 \\
\hline
\end{tabular}

Table 2: Key parameters of UPM machine base prototype used for simulations

To evaluate $\left\|H_{F y}\right\|_{\infty}$, we use the parameters in Table 2 (with $m=834 \mathrm{~kg}$ and $I=77 \mathrm{~kg}$ $\mathrm{m}^{2}$ ) to calculate $\rho=303.9 \mathrm{~mm}, \tilde{h}_{F}=0.43, \varepsilon=0.87, \zeta_{y}=0.06, \zeta_{\theta}=0.22$. The ratio $C_{\theta} / C_{y}=$ 0.39 , implying that $\left|H_{y, F y}\right|$ has the dominant peak for the decoupled system. Figure 2 reveals that $T_{y}>0$; therefore placing the isolators below the CG (i.e., $h<0$ ) will reduce the peak of 
$\left|H_{y, F y}\right|$. This is confirmed by Figure 17(a) which depicts the simulated variation of the peaks of $\left|H_{y, F y}\right|$ and $w\left|H_{\theta, F y}\right|$ as functions of $h$. For evaluating $\left\|H_{F z}\right\|_{\infty}, m=830 \mathrm{~kg}$ and $I=80 \mathrm{~kg}-\mathrm{m}^{2}$. Therefore, $\rho=310.5 \mathrm{~mm}, \tilde{r}=0.382$ and $\varepsilon=0.85, \zeta_{y}=0.06$ and $\zeta_{\theta}=0.22$. From Figure 5, $T_{\mathrm{z}}<0$ (i.e., a local maximum occurs at $h=0$ ); selecting $h<0$ or $h>0$ will reduce the peak of $\left|H_{F z}\right|$. Figure 17(b) attests to this fact.

Transmissibility (i.e., $\sigma_{H}^{2}$ ) is evaluated using the same parameters applied to $\left\|H_{F y}\right\|_{\infty}$; therefore $\tilde{h}_{w}=0.72 . T_{t r}>0$ for any value of $\varepsilon<1$ so $h<0$ is favorable for vibration reduction, as confirmed by Figure 17(c). We therefore have the very desirable condition where, compared to the recommended practice of decoupling, weak mode coupling (with $h$ $<0)$ is predicted to simultaneously reduce $\left\|H_{F y}\right\|_{\infty},\left\|H_{F z}\right\|_{\infty}$ and $\sigma_{H}^{2}$. In the rest of this section, we validate this finding experimentally by comparing residual vibration and transmissibility responses for $h=0$ (decoupled) and $h=-65 \mathrm{~mm}$ (coupled).

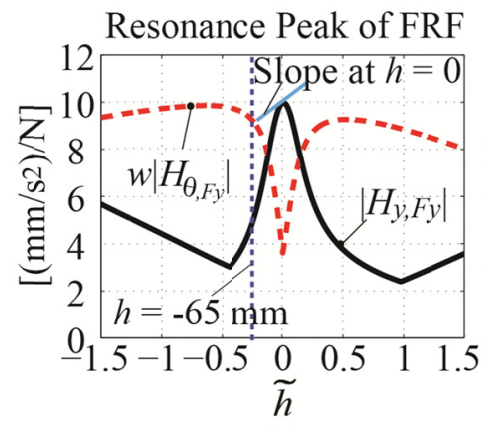

(a)

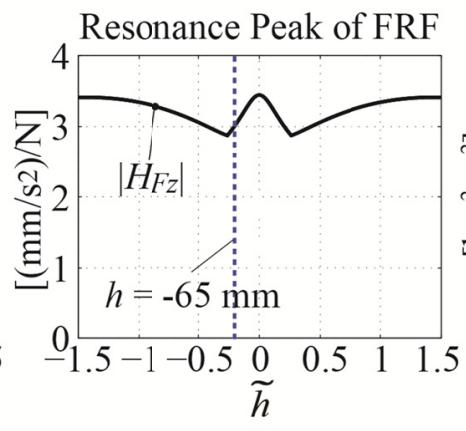

(b)

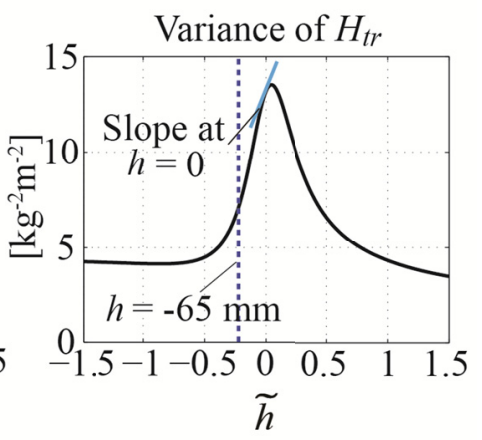

(c)

\section{Figure 17: Simulated plots of (a) $\left|H_{y, F y}\right|$ and $w\left|H_{\theta, F y}\right|$, (b) $\left|H_{F z}\right|$, and (c) variance of $H_{t r}$ of UPM base prototype as functions of dimensionless isolator height. $w=1 \mathrm{~m}$}

Figure 18 (a) and (b) show the simulated and experimentally measured $\left|H_{y, F y}\right|$ for the decoupled and coupled systems. Both are in close agreement and show about $40 \%$ reduction in the resonance peak due to mode coupling. A similar comparison of simulated 
and measured FRFs is presented in Figure 18 (c) and (d) for residual vibrations in the $z$ direction, showing about $25 \%$ reduction in the resonance peak. The effect of the reported resonance peak reductions can be seen in Figure 19 which shows the residual vibration response of the base to a constant acceleration ( $1 g)$ motion command applied to the shaker to move it from rest to $2590 \mathrm{~mm} / \mathrm{min}$. The settling time of the coupled system is significantly improved relative to the decoupled.

Figure 20 (a) and (b) compare the simulated and experimentally measured $\left|H_{t r}\right|$ of the machine. A close agreement is obtained between both plots except for very low frequencies $(<4 \mathrm{~Hz})$ where the experiment results are poor due to the inability of the hammer to properly excite the foundation. However, the benefit of mode coupling is very evident in the reduction of the resonance peaks by about $50 \%$. Moreover, attenuation beyond resonance is not adversely affected by coupling. As a final note, the conditions for maximum sensitivity for the UPM machine prototype are calculated as $\varepsilon_{y}^{*}=0.71$ or $\varepsilon_{y}^{*}=$ $1.18, \varepsilon_{z}^{*}=0.95$ and $\varepsilon_{t r}{ }^{*}=0.65$ or $\varepsilon_{t r}=1.24$. Therefore, there is some room for improvement in each response, if desired. However, one would have to decide on which response to maximize, since the conditions for maximum sensitivity are not the same for all three responses. 


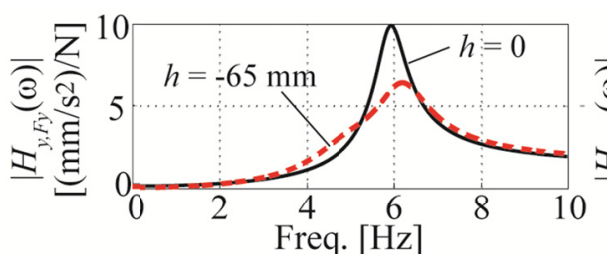

(a)

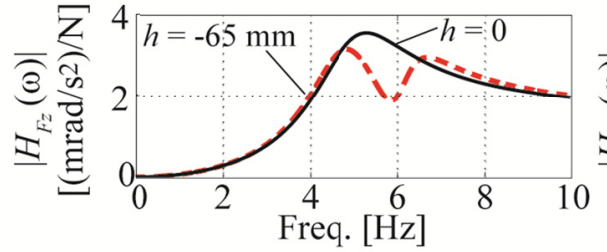

(c)

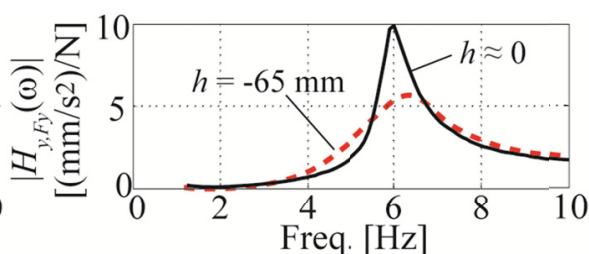

(b)

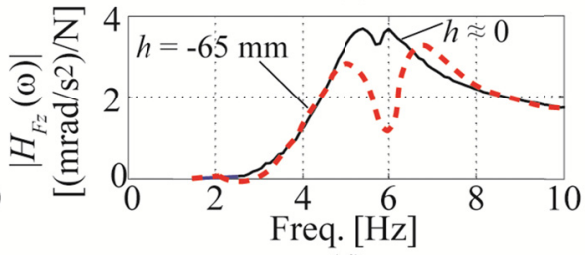

(d)

Figure 18: $\left|H_{y, F y}\right|$ obtained from (a) simulations and (b) experiments; $\left|H_{F z}\right|$ obtained from (c) simulations and (d) experiments for $h=0$ and $h=-65 \mathrm{~mm}$

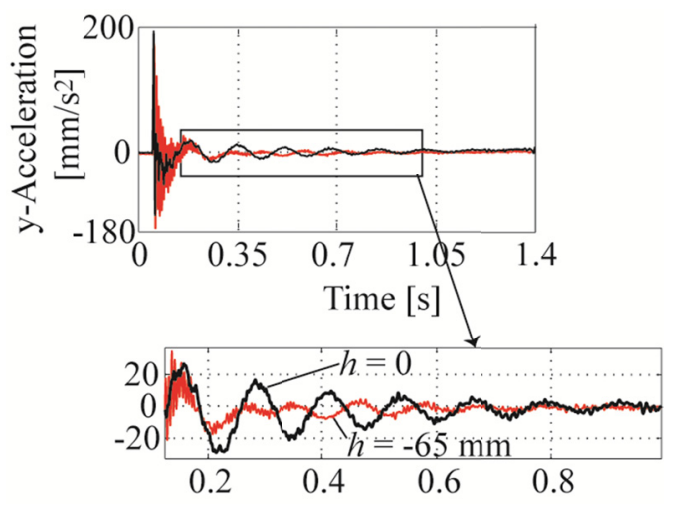

Figure 19: $y$-Acceleration of base measured at point $P$ (in Figure 16) for $h=0$ and $h=$ $-65 \mathrm{~mm}$ during motion of $y$-axis from rest to $2590 \mathrm{~mm} / \mathrm{min}$

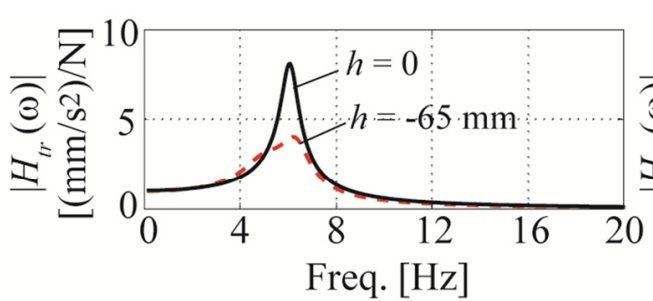

(a)

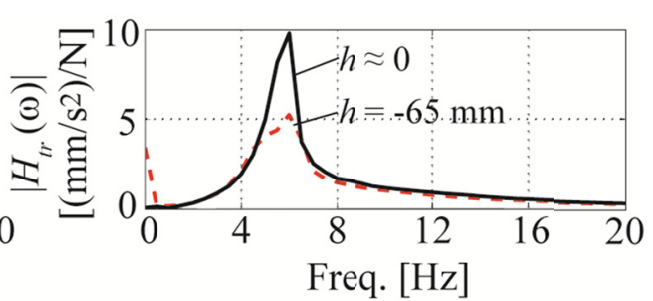

(b)

Figure 20: $\left|H_{t r}\right|$ obtained from (a) simulations and (b) experiments for $h=0$ and $h=$ $-65 \mathrm{~mm}$. 


\section{Conclusion}

This paper has investigated the effects mode coupling on the residual vibrations and ground vibrations (i.e., transmissibility) of passively-isolated UPM machines with nonproportional (NP) damping. It complements a prior investigation by the authors based on the modal damping assumption. A perturbation technique is used to analyze the $H_{\infty}$ and $H_{2}$ norms of residual vibration and transmissibility FRFs, respectively. The results show that, even though NP damping changes the vibration behavior of the machine base compared to modal damping, mode coupling still provides ample opportunities to reduce base vibrations, providing that the machine is properly designed. Guidelines for properly designing a UPM machine to best exploit mode coupling for base vibration reduction are provided. Simulations and experiments on UPM machine prototypes are used to demonstrate significant benefits of mode coupling relative to decoupling with regard to both residual vibration and transmissibility. Future work will extend the investigation to non-planar isolation systems and strong mode coupling.

\section{Acknowledgments}

The authors would like to thank Mr. Wei Hon Yap and Dr. Amirhossein Ghasemi for their assistance with some of the experimental work reported in this paper. DTL Corporation (Mori Seiki) is also appreciated for providing their 5-axis ultra-precision milling machine (NN1000 DCG) for simulations and experiments. This work is sponsored by the National Science Foundation's Grant \# 1232915. 


\section{References}

[1] Alting L, Kimura F, Hansen HN, Bissacco G. Micro engineering. Ann CIRP 2003;52(2):635-57.

[2] DeBra DB. Vibration isolation of precision machine tools and instruments. Ann CIRP 1992;41(2):711-18.

[3] Rivin EI. Vibration isolation of precision equipment. Prec Eng 1995;17:41-56.

[4] Rivin EI. Vibration isolation of precision objects. Sound and Vib 2006;40(7):12-20.

[5] Kim C-J, Oh J-S, Park C-H. Modelling vibration transmission in the mechanical and control system of a precision machine. CIRP Annals-Manufacturing Technology 2014;63:349-352.

[6] Subrahmanyari PK, Trumper DL. Synthesis of passive vibration isolation mounts for machine tools - a control systems paradigm. Proc American Control Conf 2000;4: 2886-91.

[7] Jones SD, Ulsoy AG. An approach to control input shaping with application to coordinate measuring machines. Trans ASME J Dyn Syst, Meas Contr $1999 ; 121(2): 242-7$

[8] Zuo L. Element and system design for active and passive vibration isolation. Diss. Massachusetts Institute of Technology; 2004.

[9] Heertjes M, van de Wouw N. Nonlinear dynamics and control of a pneumatic vibration isolator. J Vib and Acoustics 2006;128(4):439-448.

[10] Racca SR. How to Select Power-Train Isolators for Good Performance and Long Service Life. SAE Tech Paper Series 821095; 1982.

[11] Piersol A, Paez T. Harris' Shock and Vibration Handbook $6^{\text {th }}$ Edition. McGraw-Hill. 2010;39.13-39.14.

[12] Braun SG, Ewins DJ, Rao SS. Encyclopedia of Vibration. Elsevier; 2002, p. 14901491.

[13] Andrews F. Items which can compromise vibration isolation. http://adopt.arcetri.astro.it/html/secLBT/datasheets/vibration_isolators/Fabreeka/practical_considerations.pdf. Accessed on $10 / 9 / 2014$

[14] Technical Manufacturing Corporation, Precision Vibration Isolation Systems: Technical Background, http://www.techmfg.com/pdf/TMC\%20Techical\%20Background\%202011.pdf Accessed on 10/9/2014.

[15] Ryaboy VM. Static and dynamic stability of pneumatic vibration isolators and systems of isolators. J Sound and Vib 2014;333:31-51. 
[16] Okwudire CE, Lee J. Minimization of the Residual Vibrations of Ultra-Precision Manufacturing Machines via Optimal Placement of Vibration Isolators. Prec Eng 2012;37(2):425-435.

[17] Okwudire CE, Lee J. Optimal Motor Location for the Reduction of Residual Vibrations in Mode-Coupled Ultra-Precision Manufacturing Machines. ASME 2013 International Manufacturing Science and Engineering Conference; 2013.

[18] Okwudire CE, Kim C-J, Kim J. Reduction of the Vibrations of Ultra-precision Manufacturing Machines via Mode Coupling. ASPE 2012 Annual Meeting; 2012 October 21-26; San Diego, CA.

[19] Okwudire CE. A study on the effects of isolator, motor and work surface heights on the vibrations of ultra-precision machine tools. Proc ICOMM, Evanston, Illinois, March 2012. 31-36

[20] Anderson PW. Absence of diffusion in certain random lattices. Phys Rev 1958;109(5):1492-505.

[21] Hodges CH. Confinement of vibration by structural irregularity. J Sound and Vib 1982;82(3):411-424.

[22] Pierre C. Weak and strong vibration localization in disordered structures: A statistical investigation. J Sound and Vib 1990;139:111-132.

[23] Rajagopal G. Optimal mode localization in disordered, periodic structures. Ph.D thesis, Massachusetts Institute of technology; 1995.

[24] Igusa T. Critical configurations of systems subjected to wide-band input. J Sound and Vib 1993;168(3):525-41.

[25] Thiruvenkatanathan P, Woodhouse J, Yan J, Seshia AA. Manipulating vibration energy confinement in electrically coupled microelectromechanical resonator arrays. J Microelectromechanical Syst 2011;20:157-64.

[26] Perkins NC, Mote Jr CD. Comments on curve veering in eigenvalue problems. J Sound and Vib 1986;106(3):451-63.

[27] Balmes E. High modal density, curve veering, localization: A different perspective on the structural response. J Sound and Vib 1993;161(2):358-363.

[28] Vijayan K, Woodhouse J. Shock amplification, curve veering and the role of damping. J Sound and Vib 2013;333(5):1379-89

[29] Lee J, Okwudire CE. Optimal Damping for the Reduction of Residual Vibrations in Ultra-Precision Manufacturing Machines. ASPE 2013 Annual Meeting; 2013 October 20-25; St. Paul, MN.

[30] Lee J, Okwudire CE. Effects of Non-Proportional Damping on the Residual Vibrations of Mode-Coupled Ultra-Precision Manufacturing Machines. ASME 2014 Dynamic Systems and Control Conference; 2014 October 22-24; San Antonio, TX. 
[31] Erin C, Wilson B, Zapfe J. An improved model of a pneumatic vibration isolator: theory and experiment. J Sound and Vib 1998;218:81-101.

[32] Lee J-H, Kim K-J. A method of transmissibility design for dual-chamber pneumatic vibration isolator. J Sound and Vib 2009;323:67-92.

[33] Bartel DL, Krauter AI. Time domain optimization of a vibration absorber. J Eng Indust 1971;93(3):799-803.

[34] Kozak K, Singhose W, Ebert-Uphoff I. Performance measures for input shaping and command generation. J Dyn Sys Measurement and Cont 2006(3);128:731-736.

[35] Wang YZ, Cheng SH. The optimal design of dynamic absorber in the time domain and the frequency domain. Appl Acoust 1989;28:67-78.

[36] Ewins DJ. Modal testing: theory, practice, and application. Research Studies Press; 2000, p. 62-63.

[37] Zuo L, Nayfeh S A. The Two-Degree-of-Freedom Tuned-Mass Damper for Suppression of Single-Mode Vibration under Random and Harmonic Excitation. J Vib and Acoustics 2006;128:56-65.

[38] Asami T, Nishihara O, Baz A M. Analytical Solutions to $\mathrm{H}_{\infty}$ and $\mathrm{H}_{2}$ Optimization of Dynamic Vibration Absorbers Attached to Damped linear systems. J Vib and Acoustics 2002;124(2):284-295.

[39] Pierre C, Philip D Cha. Strong mode localization in nearly periodic disordered structures. AIAA J 1989;27(2):227-241.

[40] Pierre C. Mode phenomena and eigenvalue loci veering. J Sound and Vib 1988;126(3):485-502.

\section{Appendix}

The dimensionless $h_{N i}, I_{q i}, \omega_{n i}$ and $\omega$ (discussed in the body of the paper) are defined as

$$
\tilde{h}_{N i} \triangleq \frac{h_{N i}}{\rho} ; \tilde{I}_{q i} \triangleq \frac{I_{q i}}{I} ; \tilde{\omega} \triangleq \omega / \sqrt{k_{y} / m} ; \tilde{\omega}_{n i} \triangleq \omega_{n i} / \sqrt{k_{y} / m}
$$

where $\rho=(I / m)^{1 / 2}$ is the centroidal radius of gyration of the machine. Note that the tilde (i.e.,

$\sim$ ) accent on the dimensionless variables is omitted in the body of the paper (except for $h$ and $h_{F}$ ) to simplify the notation. The dimensionless modal parameters can be described by the functions [16] 


$$
\begin{aligned}
& \tilde{\omega}_{n i}=\sqrt{\frac{1+\varepsilon^{2}+\tilde{h}^{2} \mp \sqrt{\left(1+\varepsilon^{2}+\tilde{h}^{2}\right)^{2}-4 \varepsilon^{2}}}{2}} ; \\
& \tilde{h}_{N i}=\frac{1}{\tilde{h}}\left(\varepsilon^{2}+\tilde{h}^{2}-\tilde{\omega}_{n i}{ }^{2}\right) ; \tilde{I}_{q i}=\tilde{h}_{N i}^{2}+1
\end{aligned}
$$

Figure 21 to Figure 23 show the behavior of each variable as functions of dimensionless $h$ for various combinations of $m, I, k_{y}$ and $k_{\theta}$ (as embodied in $\varepsilon$ ). The behavior of each parameter can be grouped into two characteristic cases: $\varepsilon<1$ (i.e. $k_{\theta} I<$ $k_{y} / m$ ) and $\varepsilon>1$ (i.e. $\left.k_{\theta} I>k_{y} / m\right) . \varepsilon=1$ is a special (degenerate) situation [40] that can very rarely (if ever) be achieved in practice so it does not merit much attention.

From Figure 21, we see that as $h$ increases positively or negatively, $\omega_{n 1}$ reduces while $\omega_{n 2}$ increases monotonically. At $h=0, \omega_{n 1}$ and $\omega_{n 2}$ come closest to each other and then suddenly veer off with large curvatures without crossing. Crossing occurs only in the special case when $\varepsilon=1$ and $h=0$. Notice that as $\varepsilon$ deviates from unity, the distance between $\omega_{n 1}$ and $\omega_{n 2}$ at $h=0$ increases. According to Fig. 10, when $\varepsilon<1$, Mode 1 starts out at $h=0$ as a purely rotational mode about the CG (i.e., $\left.h_{N 1}=0\right)$. As $h$ increases positively, $h_{N 1}$ increases monotonically while for negative $h$, it decreases monotonically. Conversely, when $\varepsilon>1$, Mode 1 is a purely translational mode (i.e., $h_{N 1}=\infty$ ) at $h=0$. As $h$ increases positively, $h_{N 1}$ rapidly decreases from $+\infty$ until $h=\sqrt{ }\left(\varepsilon^{2}-1\right)$ when it suddenly changes direction and starts to increase. For negative $h$, the same trend is seen in $h_{N 1}$ but its sign is reversed. It can also be seen from Figure 22 that $h_{N 2}$ has a behavior that is qualitatively opposite to that of $h_{N 1}$ for both $\varepsilon<1$ and $\varepsilon>1$. For instance, except at $h=0, h_{N 1}$ always has the same sign as $h$ while $h_{N 2}$ always has a sign opposite to that of $h$. Figure 23 shows that the modal inertias $I_{q i}$ follow a similar trend as the node heights, except that their behaviors 
are symmetrical about $h=0$. The drastic behaviors of $\omega_{n i}, h_{N i}$ and $I_{q i}$ as functions of $h$ are related to closely-linked and well-studied phenomena known as eigenvalue curve veering and mode localization [24][40].

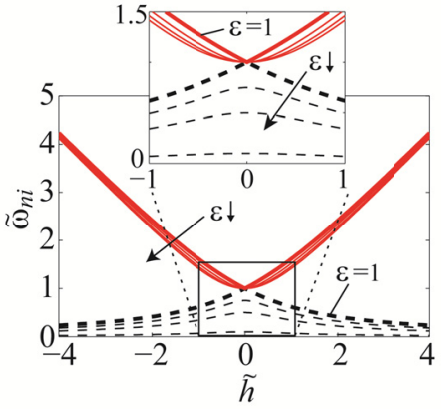

(a) $\varepsilon \leqslant 1$

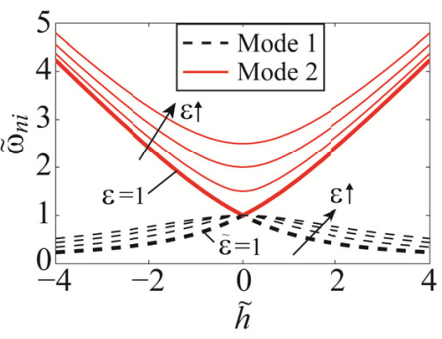

(b) $\varepsilon \geqslant 1$

Figure 21: Variation of dimensionless natural frequencies as functions of dimensionless isolator height for values of $\varepsilon$

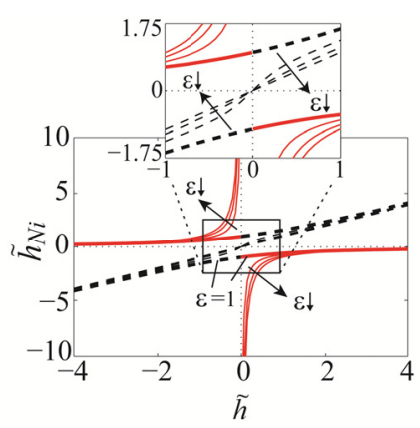

(a) $\varepsilon \leqslant 1$

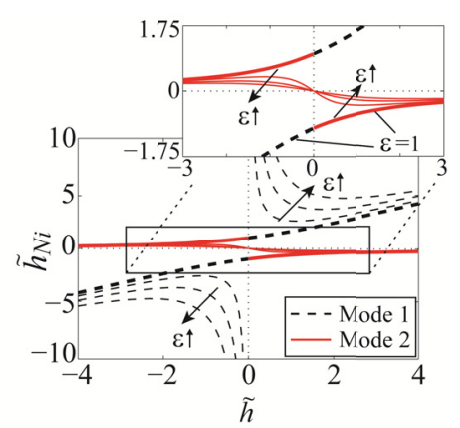

(b) $\varepsilon \geqslant 1$

Figure 22: Variation of dimensionless node height as functions of dimensionless isolator height for values of $\varepsilon$ 


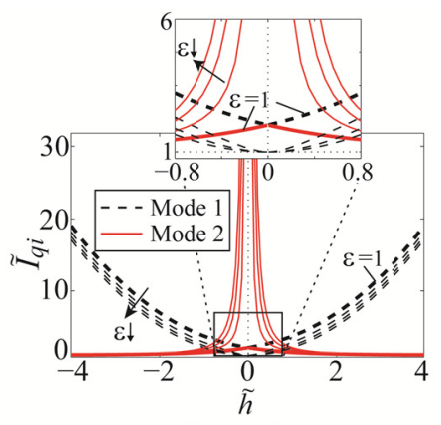

(a) $\varepsilon \leqslant 1$

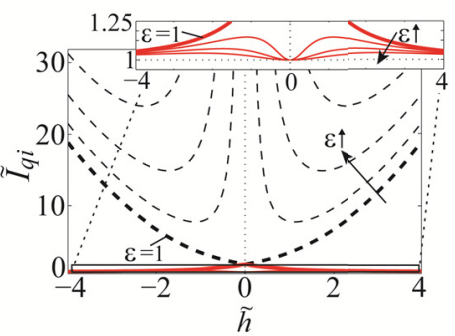

(b) $\varepsilon \geqslant 1$

Figure 23: Variation of dimensionless modal inertias as functions of dimensionless isolator height for values of $\varepsilon$ 